\title{
Cytotoxicity, Acute and Sub-chronic Toxicities of the Fruit Extract of Tetrapleura Tetraptera (Schumm. \& Thonn.) Taub. (Fabaceae)
}

Idrios N. Bonsou

University of Dschang

Armelle T. Mbaveng

University of Dschang

Gaëlle S. Nguenang

University of Dschang

Godloves F. Chi

University of Buea

Thomas Efferth

Johannes Gutenberg University

Victor Kuete ( $\square$ kuetevictor@yahoo.fr)

University of Dschang

\section{Research Article}

Keywords: Cytotoxicity, Fabaceae, spice, Tetrapleura tetraptera, Toxicity.

Posted Date: February 24th, 2022

DOI: https://doi.org/10.21203/rs.3.rs-1337474/v1

License: (9) (i) This work is licensed under a Creative Commons Attribution 4.0 International License.

Read Full License 


\section{Abstract \\ Background}

Tetrapleura tetraptera is a medicinal spice traditionally used to treat cancer, diabetes, and several other ailments. This study analyzed the cytotoxicity of the dichloromethane methanol extract of $T$. tetraptera fruits (TTF) and its constituents. The toxicity profile of the TTF extract was also evaluated in rats.

\section{Methods}

The Cytotoxicity of this extract was evaluated using the resazurin reduction assay (RRA). Acute and subchronic toxicity studies were performed according to the protocol described by the Organisation for Economic Cooperation, and Development (OECD). Hematological, serum, and urine biochemical parameters, as well as histological sections of the liver and kidney, were also evaluated based on standard methods.

\section{Results}

The TTF extract, compound 5 , and the reference drug doxorubicin were active in all 9 tested cancer cell lines. The recorded $\mathrm{IC}_{50}$ ranged from $18.32 \mu \mathrm{M}$ (against B16-F1 murine melanoma cells) to $36.18 \mu \mathrm{M}$ (against SKMel-505 BRAF wildtype melanoma cells) for TTF, from 10.02 $\mu \mathrm{M}$ (towards MaMel-80a BRAFV600E homozygous mutant melanoma cells) to $31.73 \mu \mathrm{M}$ (against SKMel-28 BRAF-V600E homozygous mutant melanoma cells) for compound 5, and from $0.22 \mu \mathrm{M}$ (against B16-F1 cells) to $9.39 \mu \mathrm{M}$ (against SKMel-505 cells) for doxorubicin. The study of acute toxicity test showed that the lethal dose $\left(\mathrm{LD}_{50}\right)$ of this extract was greater than $5000 \mathrm{mg} / \mathrm{kg}$ body weight. In the sub-chronic toxicity studies, variations were observed in some biochemical parameters, especially at higher doses.

\section{Conclusion}

TTF and its most active compound (5) are found to be potential cytotoxic agents, meanwhile, TTF was safe when given a single oral dose of $5000 \mathrm{mg} / \mathrm{kg}$. However, caution is necessary in case of prolonged oral administration due to potential alterations of renal function at high doses (> $1000 \mathrm{mg} / \mathrm{kg}$ ).

\section{Background}

Cancer remains a global health challenge with about 19.3 million new cases and 10 million deaths recorded in 2020 [1]. In many countries, cancer's rising prominence as a leading cause of death partly reflects marked declines in mortality rates of stroke and coronary heart disease compared to cancer, [1]. However, the toxicity of anticancer drugs as well as the chemoresistance constitute major challenges in 
cancer chemotherapy [2-4]. Several concepts are therefore being explored with medicinal food plants as one of the most promising ones [5-7]. Food plants commonly used in Cameroon are endowed with very good antibacterial, anticancer, antidiabetic, antiparasitic, and antiviral activities due to their richness in secondary metabolites [8-16].

Tetrapleura tetraptera is a widely distributed plant in West Africa. It is commonly known as Prekese and Aridan in Ghana and Nigeria, respectively, where it is highly valued for its use in traditional medicine and its nutritional properties [17]. It is a perennial plant belonging to the Fabaceae family, also found in the West Cameroon region, where it is traditionally used as a plant of choice in the treatment of obesity [18]. T. tetraptera contains several secondary metabolites belonging to different classes such as flavonoids, alkaloids, phenolic compounds, tannins, and saponins [19]. The ethanolic extract of $T$. tetraptera fruits (TTF) inhibited the proliferation of carcinoma cell lines in vitro and prolonged the life of albino mice by reducing tumor cell viability and tumor size in vivo [20]. Furthermore, the methanol crude extract of TTF fruits and four of its isolated constituents had antiproliferative activity towards drug-susceptible and multidrug-resistant cancer lines, with $\mathrm{IC}_{50}$ values for the crude extract and the most active compound (olean-12-en-3- $\beta$ - $O_{-D}$-glucopyranoside) ranging from $10.27 \mu \mathrm{g} / \mathrm{mL}$ (in CCRF-CEM leukemia cells) and $23.61 \mu \mathrm{g} / \mathrm{mL}$ (against HCT116 p53/-/- colon adenocarcinoma cells) and from $4.76 \mu \mathrm{M}$ (against CCRFCEM cells) to $12.92 \mu \mathrm{M}$ (against HepG2 hepatocarcinoma cells) [21]. The cytotoxicity of botanicals from this plant was also reported against Jurkat leukemia cells and MCF-7 breast cancer cells [22]. The hydroethanolic extract of TTF had lipid-lowering, anti-inflammatory, and hypoglycemic activity, justifying its use in the management of obesity and type 2 diabetes [23]. The ethanolic extract of TTF and the aqueous extract of its bark induced renal toxicity and an increase in LDL-cholesterol, as well as liver toxicity at high doses of $400 \mathrm{mg} / \mathrm{kg}$ [24]. However, very little information is available on the toxicity of the dichloromethane-methanol extract of the fruits of this plant that previously showed cytotoxic effects [21]. The current study aimed at further evaluating the antiproliferative activity of TTF on a wider panel of resistant and metastatic lines and determining the toxicological profile of the active extract.

\section{Methods}

\section{Collection and identification of plant material}

Following the approval of our research project by the University of Dschang (Faculty of Science), the plant fruits were afforded in the Dschang locality in March 2021 (Subdivision of Cameroon, Western Region, $5^{\circ} 27^{\prime} \mathrm{N} / 10^{\circ} 04^{\prime} \mathrm{E}$ ). No authorization to collect the plant sample was needed. A sample of the plant consisting of leaves and fruits was then placed in the Cameroun National Herbarium (NHC) located in Yaoundé. The sample was identified and authenticated by Mr NANA Victor as Tetrapleura tetraptera (Schumm. \& Thonn.) Taub in comparison with the specimen of the herbarium under the voucher number 19785 SRF/Cam.

\section{Preparation of dichloromethane methanolic crude extract of T. tetraptera fruits}


The fruits of $T$. tetraptera were dried and powdered, then macerated using dichloro-methane methanol $\left(\mathrm{CH}_{2} \mathrm{Cl}_{2}-\mathrm{MeOH} ; 1: 1\right)$ for $48 \mathrm{~h}$ at room temperature (RT). The macerate was further filtrated with Whatman paper No. 1. The solvent was removed from the filtrate by a rotary evaporator (BÜCHI R-200) at $65^{\circ} \mathrm{C}$ to afford the crude extract (TTF). To completely remove the residual solvent, TTF was further dried in an oven at $40^{\circ} \mathrm{C}$ for $6-8 \mathrm{~h}$ and stored in a refrigerator at $4^{\circ} \mathrm{C}$ for further uses.

\section{Phytochemicals and chemicals}

The experimented phytochemicals were: (3R, 4S)-3,4-dimethyloxetan-2-one (1), luteolin (2), stigmasterol (3), 3-O-[6'-O-undecanoyl- $\beta_{-D}$-glucopyranosyl]stigmasterol (4), oleanan-12-en-3- $\beta-O_{-D}$-glucopyranoside(5),3-

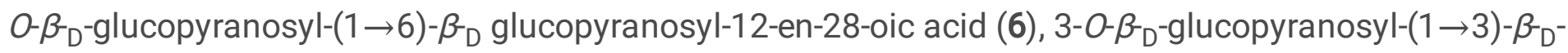
glucopyranosyl-27-hydroxyolean-12-ene-28-oic acid (7), methyl-0- $\beta_{D_{D}}$-glucopyranoside (8) and $\beta_{D^{-}}$ fructofuranosyl-(2 $\rightarrow 1)-\beta_{D}$-glucopyranoside (9) (Fig. 1). Their isolation and identification from the dichloromethane-methanol (1:1) extract of TTF are given within the extra Supplementary file. Doxorubicin (purity: $98.0 \%$ ) purchased from Sigma-Aldrich (Munich, Germany) was obtained from the Johannes Gutenberg University Medical Center (Mainz, Germany) [21].

\section{Investigated cell lines and their origin}

The nine (9) malignant cell lines utilized in this study enclosed in each human and animal-originated cells. Human melanoma cell lines enclosed MaMel-80a, SKMel-28 and A2058 were used as human melanoma highly lymphoid tissue metastatic cell line [25]; Mel-2a and SKMel-505 human melanoma cell line derived from the metastatic site; MV3 human melanoma cells from lung metastases in nude mice [26]; the animal originated cell line enclosed CC531 rat tumor colorectal cell line [27]; B16-F1 and B16-F10 a murine melanoma cell lines from a C57BL/6J mouse [28-30]. SkMel-28, MaMel-80a, Mel-2A, and MV3 cell lines were provided by Prof. David Schrama (Department of Dermatology, Julius-Maximilians University, Würzburg, Germany); B16-F10 and B16-F1 murine melanoma cell lines were provided by Prof. Ugur Sahin (TRON-Translational Oncology at the University Medical Center of Johannes Gutenberg University $\mathrm{GmbH}$, Mainz, Germany); CC531 rat colon adenocarcinoma cell line was purchased from CLS Cell Lines Service GmbH (Eppelheim, Germany), A2058 and SK-Mel505 human melanoma cell lines provided by Dr. Wynand P. Roos (Institute of Toxicology, Medical University Center, Mainz, Germany).

\section{Cytotoxicity testing of the botanical, phytochemicals, and doxorubicin by resazurin reduction assay (RRA)}

To assess the actions of TTF extract, phytochemicals (1-9), and doxorubicin on cellular multiplication, the well-described RRA was applied as said earlier [31-32] at experimental conditions like those formerly published [33-36]. The botanical (TTF) and phytochemicals were tested at concentrations starting from $0.32-40 \mu \mathrm{g} / \mathrm{mL}$ and $0.78-100 \mu \mathrm{M}$, respectively. Cells were incubated for $72 \mathrm{~h}$ in humidified $5 \% \mathrm{CO}_{2}$ atmosphere at $37^{\circ} \mathrm{C}$, and also the fluorescence was measured with Infinite M2000 ProTM plate reader (Tecan, Crailsheim, Germany) at $544 \mathrm{~nm}$ as excitation wavelength and $590 \mathrm{~nm}$ as emission wavelength. 
The sample's concentrations needed to inhibit $50 \%$ of the cell proliferation portrayed their $I_{50}$ values and were deduced from a calibration curve by linear regression using Microsoft Excel 2007 [32].

\section{Experimental animals}

For the toxicological study of the crude extract of TTF, young healthy males, and female albino Wistar rats, nulliparous and non-pregnant, aged between 1.5-2 months and weighing between 120 and $140 \mathrm{~g}$, respectively, were used. The animals were obtained from the Animal House of the Department of Biochemistry, University of Dschang. They were maintained in an animal room at a temperature of $20 \pm$ $2^{\circ} \mathrm{C}$ under a standard animal room condition of $12 \mathrm{~h}$ light/dark cycle and fed with a standard food [37] and received drinking water ad libitum. The animals were individualized one week before the start of the experiment. The experimental protocols used for the present work were designed in concordance with the internationally accepted standard ethical guidelines for laboratory animals use and care as described in the guidelines of the European Union Institutional Ethics Committee on Animal Care (Council EEC 86/609/EEC of the 24th November 1986) and were approved by the Local Ethical Committee of the Faculty of Science (University of Dschang - Cameroon). All sections of this report comply with ARRIVE Guidelines for Reporting Animal Research [38].

\section{Acute oral toxicity study}

Acute toxicity assessment of the dichloromethane methanol crude extract of TTF was carried out according to the experimental protocol proposed by OECD Guideline 425 [39]. We performed the test at the single dose of $5000 \mathrm{mg} / \mathrm{kg} \mathrm{BW}$. We had two experimental groups of albinos female Wistar rats, a test group $(n=5)$ and a control group $(n=5)$. After one week of acclimatisation, the animals were given a single dose of $5000 \mathrm{mg} / \mathrm{kg}$ BW of the crude extract of T. tetraptera. Afterward, administration of the extract by gavage, the behavior of the test animals was carefully observed for a period of $4 \mathrm{~h}$ to detect any signs of toxicity. After $48 \mathrm{~h}$ observation, the $\mathrm{LD}_{50}$ was determined, and the animals were then left for observation for an additional period of 14 days before being sacrificed for macroscopic observation of various organs.

\section{Study of sub-chronic oral toxicity}

The sub-chronic toxicity was carried out on a total of 32 albino rats belonging to both genders, males, and females. The animals were divided into four groups of eight animals each, i.e., four male and four female rats. The first group was the control and received the vehicle consisting of a $5 \%$ dimethylsulfoxide (DMSO) solution, while the other three groups constituted the test groups and respectively received a repeated administration by gavage of 250,500 , and $1000 \mathrm{mg} / \mathrm{kg} \mathrm{BW}$ of the dichloromethane methanol crude extract of TTF for a period of 28 days. The animals were weighed every other day, the food intake was taken daily, and the water was renewed. The animals were carefully observed every day for any changes in clinical signs or even death. On the last day of gavage, the rats were fasted for a period of 12 $\mathrm{h}$ before being sacrificed by intraperitoneal injection of ketamine. The blood was then collected by cardiac puncture and introduced into two different types of tubes, namely dry tubes for obtaining serum for the determination of biochemical parameters, and ethylenediaminetetraacetic acid (EDTA) tubes for 
the determination of haematological parameters. Organs such as heart, liver, kidney, and spleen were collected for determination of their relative weights. Those heavily involved in xenobiotic metabolism and elimination such as liver and kidney were used for histological sections [39].

\section{Hematological parameters}

Blood samples collected by cardiac puncture and stored in EDTA tubes were used to perform hematological parameters by a blood count using an impedance hematology machine (QBC Auto-read Plus, United Kingdom). The parameters analyzed included red blood cells (RBC), red blood cell distribution width (CV), and red blood cell distribution width (SD), hemoglobin ( $\mathrm{Hb})$, mean corpuscular volume (MCV); mean corpuscular hemoglobin ( $\mathrm{MCH})$, mean corpuscular hemoglobin concentration $(\mathrm{MCHC})$, leukocytes, neutrophils, eosinophils, basophils, lymphocytes, and monocytes count, platelets (PLT), hematocrit (HCT).

\section{Biochemical parameters}

Blood samples were collected into dried tubes and allowed to stand for $45 \mathrm{~min}$ at room temperature before being centrifuged at $3400 \mathrm{rpm}$ for $10 \mathrm{~min}$. The serum obtained was introduced into Eppendorf tubes and stored at $-25^{\circ} \mathrm{C}$ and then used to evaluate the following parameters: creatinine, alanine aminotransferase (ALAT), aspartate aminotransferase (ASAT), alkaline phosphatase (ALP), total protein, total cholesterol (TC), high-density lipoprotein (HDL), low-density lipoprotein (LDL), and triglyceride (TG). These parameters were assessed using standard analytical kits (Spinreact, Spain).

\section{Histological analysis}

For histological analysis, the liver and kidney from the sacrificed animals were first rinsed in a saline solution $(0.9 \% \mathrm{NaCl})$ before being introduced into 3.7\% formaldehyde for conservation. These tissues were subsequently dehydrated in increasing concentrations of alcohol $(70 \%, 90 \%$, and $100 \%)$, inserted into paraffin, and cut into sections of 4-5 $\mu \mathrm{m}$. these paraffin sections (5 $\mu \mathrm{m}$ thick) were stained with hematoxylin-eosin prior to microscopic examination [40].

\section{Statistical analysis}

Statistical analysis was performed using SPSS version 20.0 for Windows. The results were expressed as mean value \pm standard deviation (SD.) and the comparisons were performed by the analysis of variance using the analysis of variance (ANOVA) test. Differences between averages of control and drug-treated groups were separated using the Waller-Duncan test. A probability value of less than 0.05 was fixed as the statistical significance criterion [41].

\section{Results}

Cytotoxicity. In this study where we evaluated the cytotoxicity of crude extract and phytochemicals 1-9, and doxorubicin using RRA towards 9 carcinoma cancer cell lines; it was found that, the crude TTF extract, phytochemical $\mathbf{5}$, and doxorubicin had good cytotoxic effects against the nine (9) cancer cell lines 
tested. The recorded $\mathrm{IC}_{50}$ values varied from $18.32 \mu \mathrm{M}$ (against B16-F1 murine melanoma cells) to 36.18 $\mu \mathrm{M}$ (against SKMel-505 BRAF wildtype melanoma cells) for TTF, from $10.02 \mu \mathrm{M}$ (towards MaMel-80a BRAF-V600E homozygous mutant melanoma cells) to $31.73 \mu \mathrm{M}$ (against SKMel-28 BRAF-V600E homozygous mutant melanoma cells) for compound $\mathbf{5}$, and from $0.22 \mu \mathrm{M}$ (against B16-F1 cells) to 9.39 $\mu \mathrm{M}$ (against SKMel-505 cells) for doxorubicin. Eight out of 9 cell lines tested were sensitive to compound 2 with $\mathrm{IC}_{50}$ values ranging from $32.20 \mu \mathrm{M}$ (towards B16-F1 cells) to $102 \mu \mathrm{M}$ (against SKMel-505 cells). In the sensitive cancer cell lines, the $\mathrm{IC}_{50}$ values ranged from $28.67 \mu \mathrm{M}$ (against MaMel-80a melanoma cells) to $109.24 \mu \mathrm{M}$ (towards Mel-2a melanoma cells) for compound 6; and from $29.08 \mu \mathrm{M}$ (against MaMel-80a melanoma cells) to $79.42 \mu \mathrm{M}$ (against A2058 melanoma cells) for compound 7. It was noticed that compounds $\mathbf{1}, \mathbf{3}, \mathbf{4}, \mathbf{8}$, and $\mathbf{9}$ were not active above $150 \mu \mathrm{M}$. Rrecorded $\mathrm{IC}_{50}$ values are summarized in Table 1. 
Table 1

Cytotoxicity of extracts, compounds and doxorubicin towards human melanoma and other animal cancer cell lines as determined by RRA.

\begin{tabular}{|c|c|c|c|c|c|c|c|}
\hline \multirow[t]{2}{*}{ Features and cell lines } & & \multicolumn{6}{|c|}{$\mathrm{IC}_{50}$ values $(\mu \mathrm{M})$} \\
\hline & & TTF & 2 & 5 & 6 & 7 & Doxorubicin \\
\hline \multirow{2}{*}{$\begin{array}{l}\text { BRAF-V600E } \\
\text { homozygous mutant } \\
\text { melanoma }\end{array}$} & $\begin{array}{l}\text { MaMel- } \\
80 a\end{array}$ & $\begin{array}{l}30.18 \\
\pm 2.89\end{array}$ & $\begin{array}{l}44.28 \pm \\
3.29\end{array}$ & $\begin{array}{l}10.02 \\
\pm 0.21\end{array}$ & $\begin{array}{l}28.67 \\
\pm 3.12\end{array}$ & $\begin{array}{l}29.08 \\
\pm 1.48\end{array}$ & $8.66 \pm 0.56$ \\
\hline & $\begin{array}{l}\text { SKMel- } \\
28\end{array}$ & $\begin{array}{l}28.23 \\
\pm 1.72\end{array}$ & $\begin{array}{l}67.28 \pm \\
5.12\end{array}$ & $\begin{array}{l}31.73 \\
\pm 2.20\end{array}$ & $\begin{array}{l}33.95 \\
\pm 2.53\end{array}$ & $\begin{array}{l}33.11 \\
\pm 2.19\end{array}$ & $2.14 \pm 0.12$ \\
\hline \multirow{2}{*}{$\begin{array}{l}\text { BRAF-V } 600 \mathrm{E} \\
\text { heterozygous mutant } \\
\text { melanoma }\end{array}$} & A2058 & $\begin{array}{l}19.48 \\
\pm 0.82\end{array}$ & $\begin{array}{l}78.76 \pm \\
5.65\end{array}$ & $\begin{array}{l}22.77 \\
\pm 1.72\end{array}$ & $>150$ & $\begin{array}{l}79.42 \\
\pm 4.41\end{array}$ & $0.29 \pm 0.04$ \\
\hline & Mel-2a & $\begin{array}{l}18.96 \\
\pm 0.69\end{array}$ & $\begin{array}{l}102.56 \\
\pm 8.94\end{array}$ & $\begin{array}{l}25.45 \\
\pm 3.01\end{array}$ & $\begin{array}{l}109.24 \\
\pm 8.44\end{array}$ & $>150$ & $6.63 \pm 0.41$ \\
\hline \multirow[t]{2}{*}{$\begin{array}{l}\text { BRAF wildtype } \\
\text { melanoma }\end{array}$} & MV3 & $\begin{array}{l}28.72 \\
\pm 3.11\end{array}$ & $\begin{array}{l}78.33 \pm \\
6.29\end{array}$ & $\begin{array}{l}23.52 \\
\pm 1.27\end{array}$ & $>150$ & $>150$ & $7.09 \pm 0.59$ \\
\hline & $\begin{array}{l}\text { SKMel- } \\
505\end{array}$ & $\begin{array}{l}36.18 \\
\pm 2.62\end{array}$ & $>150$ & $\begin{array}{l}22.18 \\
\pm 2.01\end{array}$ & $\begin{array}{l}88.74 \\
\pm 6.42\end{array}$ & $>150$ & $9.39 \pm 1.01$ \\
\hline $\begin{array}{l}\text { Rat colon } \\
\text { adenocarcinoma }\end{array}$ & CC531 & $\begin{array}{l}18.95 \\
\pm 1.27\end{array}$ & $\begin{array}{l}54.39 \pm \\
3.63\end{array}$ & $\begin{array}{l}10.21 \\
\pm 0.14\end{array}$ & $\begin{array}{l}66.38 \\
\pm 4.77\end{array}$ & $\begin{array}{l}54.11 \\
\pm 3.10\end{array}$ & $0.44 \pm 0.23$ \\
\hline \multirow[t]{2}{*}{ Murine melanoma } & B16-F1 & $\begin{array}{l}18.32 \\
\pm 0.96\end{array}$ & $\begin{array}{l}32.20 \pm \\
2.17\end{array}$ & $\begin{array}{l}24.08 \\
\pm 0.99\end{array}$ & $\begin{array}{l}44.90 \\
\pm 5.03\end{array}$ & $\begin{array}{l}69.04 \\
\pm 4.95\end{array}$ & $0.22 \pm 0.01$ \\
\hline & $\begin{array}{l}\text { B16- } \\
\text { F10 }\end{array}$ & $\begin{array}{l}20.04 \\
\pm 1.45\end{array}$ & $\begin{array}{l}41.85 \pm \\
3.69\end{array}$ & $\begin{array}{l}24.64 \\
\pm 2.56\end{array}$ & $\begin{array}{l}50.12 \\
\pm 3.76\end{array}$ & $\begin{array}{l}61.45 \\
\pm 5.21\end{array}$ & $0.24 \pm 0.03$ \\
\hline \multicolumn{8}{|c|}{ 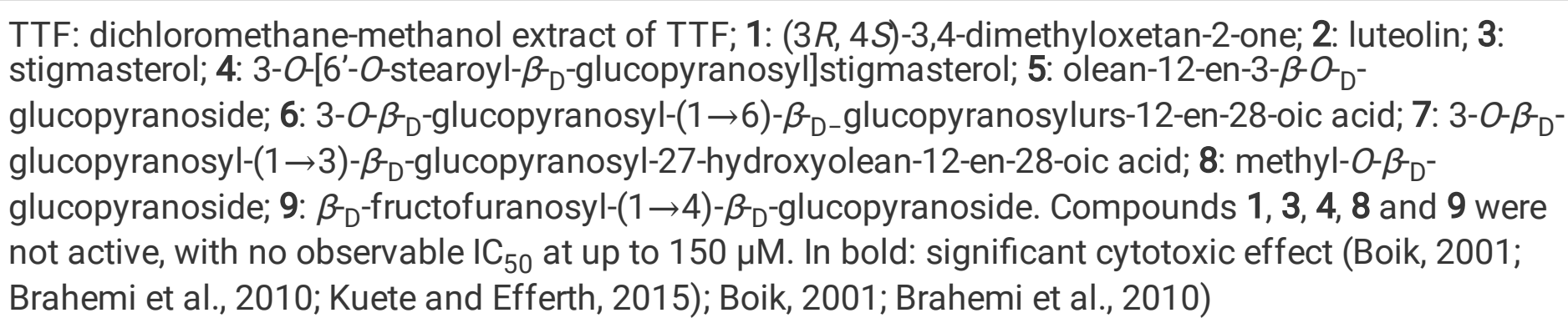 } \\
\hline
\end{tabular}

Acute toxicity of TTF. A single oral dose administration of $5000 \mathrm{mg} / \mathrm{kg}$ BW TTF in female rats did not cause any deaths $48 \mathrm{~h}$ following the administration of TTF and even during 14 days of observation. No signs of toxicity in general appearance (reduction of locomotion, stool appearance, drowsiness, salivation, reaction to noise) were observed in animals receiving this dose after 14 days of observation. Based on the OECD principle, the $\mathrm{LD}_{50}$ of the dichloromethane methanol extract of TTF was estimated to be higher than $5000 \mathrm{mg} / \mathrm{kg}$.

Effect of TTF on food consumption and body weight. Neither death nor toxicity signs were observed on animals following 28 days of treatment with various doses of $\operatorname{TTF}(250,500,1000 \mathrm{mg} / \mathrm{kg} \mathrm{BW})$. Figures 2 and 3 summarise the data of food intake and body weight evolution respectively. No significant 
differences $(p \geq 0.05)$ in food consumption were noted in treated and untreated males, while a significant decrease $(p<0.05)$ in food consumption was observed in treated females until the third week when no significant differences were observed. There was no weight loss in treated male and female animals and the control in this study. However, there was a small gain in body weight in animals treated at 500 and $1000 \mathrm{mg} / \mathrm{kg}$ in males and $500 \mathrm{mg} / \mathrm{kg}$ in females compared to the control.

Effect of TTF on relative organ weight. Table 2 summarises the relative organ weight of liver, kidney, lung, heart, and spleen for male and female rats. No significant differences $(p \geq 0.05)$ in relative liver, kidney, lung, and heart organ weights were observed in females for all dose groups tested compared to the control group, while a significant decrease in relative spleen weight was observed for all dose levels administered compared to the control group. A significant decrease $(p<0.05)$ in relative liver and spleen organ weights was noted at all doses in male rats compared to the control group. However, there was no significant change in the relative weights of the kidney, lung, and heart organs in male rats.

Table 2

Effect of different doses of T. tetrapleura extract on the relative weight of organs.

\begin{tabular}{|c|c|c|c|c|c|c|c|c|}
\hline \multirow{2}{*}{$\begin{array}{l}\text { Doses } \\
(\mathrm{mg} / \mathrm{Kg})\end{array}$} & \multicolumn{4}{|l|}{ Female } & \multicolumn{4}{|l|}{ Male } \\
\hline & 0 & 250 & 500 & 1000 & 0 & 250 & 500 & 1000 \\
\hline Liver (g) & $\begin{array}{l}3.12 \pm \\
0.09 a b\end{array}$ & $\begin{array}{l}3.21 \pm \\
0.07^{b}\end{array}$ & $\begin{array}{l}3.10 \pm \\
0.03 a b\end{array}$ & $\begin{array}{l}3.09 \pm \\
0.05^{a}\end{array}$ & $\begin{array}{l}3.39 \pm \\
0.13^{b}\end{array}$ & $\begin{array}{l}3.08^{ \pm} \\
0.02^{a}\end{array}$ & $\begin{array}{l}3.10 \pm \\
0.08^{a}\end{array}$ & $\begin{array}{l}3.06 \pm \\
0.07^{\mathrm{a}}\end{array}$ \\
\hline $\begin{array}{l}\text { Kidneys } \\
\text { (g) }\end{array}$ & $\begin{array}{l}0.65 \pm \\
0.02^{a}\end{array}$ & $\begin{array}{l}0.69 \pm \\
0.02^{a}\end{array}$ & $\begin{array}{l}0.67 \pm \\
0.04^{a}\end{array}$ & $\begin{array}{l}0.64 \pm \\
0.03^{a}\end{array}$ & $\begin{array}{l}0.65 \pm \\
0.03^{a}\end{array}$ & $\begin{array}{l}0.66 \pm \\
0.02^{a}\end{array}$ & $\begin{array}{l}0.64 \pm \\
0.03^{a}\end{array}$ & $\begin{array}{l}0.64 \pm \\
0.03^{a}\end{array}$ \\
\hline Lungs (g) & $\begin{array}{l}0.58 \pm \\
0.03 a b\end{array}$ & $\begin{array}{l}0.62 \pm \\
0.02^{b}\end{array}$ & $\begin{array}{l}0.65 \pm \\
0.07 \mathrm{ab}\end{array}$ & $\begin{array}{l}0.56 \pm \\
0.04^{a}\end{array}$ & $\begin{array}{l}0.59 \pm \\
0.03^{a}\end{array}$ & $\begin{array}{l}0.58 \pm \\
0.05^{a}\end{array}$ & $\begin{array}{l}0.61 \pm \\
0.02^{a}\end{array}$ & $\begin{array}{l}0.65 \pm \\
0.03^{a}\end{array}$ \\
\hline Heart (g) & $\begin{array}{l}0.30 \pm \\
0.01^{a}\end{array}$ & $\begin{array}{l}0.32 \pm \\
0.02^{a}\end{array}$ & $\begin{array}{l}0.31 \pm \\
0.02^{a}\end{array}$ & $\begin{array}{l}0.30 \pm \\
0.02^{a}\end{array}$ & $\begin{array}{l}0.32 \pm \\
0.02^{a}\end{array}$ & $\begin{array}{l}0.30 \pm \\
0.02^{a}\end{array}$ & $\begin{array}{l}0.30 \pm \\
0.01^{a}\end{array}$ & $\begin{array}{l}0.31 \pm \\
0.02^{a}\end{array}$ \\
\hline $\begin{array}{l}\text { Spleen } \\
\text { (g) }\end{array}$ & $\begin{array}{l}0.27 \pm \\
0.02^{b}\end{array}$ & $\begin{array}{l}0.25 \pm \\
0.02^{b}\end{array}$ & $\begin{array}{l}0.22 \pm \\
0.01 \mathrm{a}\end{array}$ & $\begin{array}{l}0.21 \pm \\
0.02^{a}\end{array}$ & $\begin{array}{l}0.30 \pm \\
0.06^{\mathrm{b}}\end{array}$ & $\begin{array}{l}0.24 \pm \\
0.01^{b}\end{array}$ & $\begin{array}{l}0.23 \pm \\
0.02 a b\end{array}$ & $\begin{array}{l}0.21 \pm \\
0.01 \stackrel{a}{ }\end{array}$ \\
\hline
\end{tabular}

Effect of TTF on hematological parameters. The results of the haematological parameters (Table 3 ) revealed an increase $(p<0.05)$ in white blood cell count at $1000 \mathrm{mg} / \mathrm{kg} \mathrm{BW}$. In addition, there was a significant decrease $(p<0.05)$ in the level of HGB, HCT in female rats receiving the $1000 \mathrm{mg} / \mathrm{kg}$ dose compared to the control group. We also observed a significant decrease $(p<0.05)$ in PLT and PCT levels for all the different doses tested compared to the control group. In male rats, a significant decrease $(p<$ 0.05 ) was observed at $1000 \mathrm{mg} / \mathrm{kg}$ for HCT and PCT levels, and at all doses tested for PLT levels. There was also a significant increase in GR and a non-significant increase in WBC compared to control. 
Table 3

Hematological parameters in male and female rats after 28 days of administration

Sexe Female Male

\begin{tabular}{lllllllll}
$\begin{array}{l}\text { Dose } \\
(\mathrm{mg} / \mathrm{kg})\end{array}$ & $\mathbf{0}$ & $\mathbf{2 5 0}$ & $\mathbf{5 0 0}$ & 1000 & $\mathbf{0}$ & 250 & 500 & 1000 \\
\hline WBC & $4.60 \pm$ & $4.87 \pm$ & $5.30 \pm$ & $5.80 \pm$ & $5.47 \pm$ & $5.67 \pm$ & $6.10 \pm$ & $6.00 \pm$ \\
$\left(10^{3} / \mu \mathrm{L}\right)$ & $0.36^{\mathrm{ab}}$ & $0.12^{\mathrm{a}}$ & $0.61^{\mathrm{bc}}$ & $0.35^{\mathrm{c}}$ & $0.31^{\mathrm{a}}$ & $0.78^{\mathrm{a}}$ & $0.66^{\mathrm{a}}$ & $0.60^{\mathrm{a}}$ \\
\hline $\mathrm{LY}(\%)$ & $65.87 \pm$ & $64.03 \pm$ & $65.50 \pm$ & $69.30 \pm$ & $65.80 \pm$ & 62.93 & $62.80 \pm$ & $62.37 \pm$ \\
& $1.76^{\mathrm{ab}}$ & $1.80^{\mathrm{a}}$ & $3.17^{\mathrm{ab}}$ & $1.91^{\mathrm{b}}$ & $4.39^{\mathrm{a}}$ & \pm 2.69 & $5.30^{\mathrm{a}}$ & $3.32^{\mathrm{a}}$ \\
& & & & & & & & \\
\hline $\mathrm{MO}(\%)$ & $6.12 \pm$ & $5.13 \pm$ & $5 \pm$ & $5.83 \pm$ & $4.37 \pm$ & $5.70 \pm$ & $6.35 \pm$ & $3.73 \pm$ \\
& $0.55^{\mathrm{a}}$ & $0.61^{\mathrm{a}}$ & $0.89^{\mathrm{a}}$ & $0.70^{\mathrm{a}}$ & $0.81^{\mathrm{ab}}$ & $0.96^{\mathrm{b}}$ & $1.2^{\mathrm{b}}$ & $0.95 \mathrm{a}$ \\
\hline $\mathrm{GR}(\%)$ & $27.80 \pm$ & $30.83 \pm$ & $30.50 \pm$ & $25.97 \pm$ & $26.80 \pm$ & 30.97 & $32.60 \pm$ & $32.20 \pm$ \\
& $1.77^{\mathrm{ab}}$ & $1.65^{\mathrm{b}}$ & $2.69^{\mathrm{b}}$ & $0.76^{\mathrm{a}}$ & $0.30^{\mathrm{a}}$ & $\pm 1.72^{\mathrm{b}}$ & $2.78^{\mathrm{b}}$ & $1.51^{\mathrm{b}}$ \\
\hline $\mathrm{RBC}$ & $8.56 \pm$ & $8.66 \pm$ & $8.69 \pm$ & $8.25 \pm$ & $9.12 \pm$ & $8.70 \pm$ & $8.50 \pm$ & $8.94 \pm$ \\
$\left(10^{6} / \mu \mathrm{L}\right)$ & $0.48^{\mathrm{a}}$ & $0.46^{\mathrm{a}}$ & $0.67^{\mathrm{a}}$ & $0.21^{\mathrm{a}}$ & $0.48^{\mathrm{a}}$ & $0.31^{\mathrm{a}}$ & $0.18^{\mathrm{a}}$ & $0.45^{\mathrm{a}}$ \\
\hline $\mathrm{HGB}$ & $18.30 \pm$ & $18.27 \pm$ & $17.70 \pm$ & $16.20 \pm$ & $17.53 \pm$ & $17.5 \pm$ & $16.50 \pm$ & $17.33 \pm$ \\
$(\mathrm{g} / \mathrm{dL})$ & $0.66^{\mathrm{b}}$ & $0.76^{\mathrm{b}}$ & $0.72^{\mathrm{b}}$ & $0.46^{\mathrm{a}}$ & $0.12^{\mathrm{a}}$ & $0.93^{\mathrm{a}}$ & $0.26^{\mathrm{a}}$ & $0.90^{\mathrm{a}}$ \\
\hline HCT (\%) & $54.43 \pm$ & $54.13 \pm$ & $51.97 \pm$ & $47.77 \pm$ & $54.57 \pm$ & 51.03 & $52.30 \pm$ & $49.47 \pm$ \\
& $1.66^{\mathrm{b}}$ & $1.56^{\mathrm{b}}$ & $2.22^{\mathrm{ab}}$ & $2.92^{\mathrm{a}}$ & $2.94^{\mathrm{b}}$ & \pm & $2.46^{\mathrm{ab}}$ & $2.02^{\mathrm{a}}$ \\
& & & & & & $1.83^{\mathrm{ab}}$ & & \\
\hline
\end{tabular}

\begin{tabular}{|c|c|c|c|c|c|c|c|c|}
\hline $\operatorname{MCV}(f L)$ & $\begin{array}{l}60.10 \pm \\
0.78^{b}\end{array}$ & $\begin{array}{l}60.43 \pm \\
1.19^{\mathrm{b}}\end{array}$ & $\begin{array}{l}59.67 \pm \\
1.07^{\mathrm{ab}}\end{array}$ & $\begin{array}{l}57.90 \pm \\
0.79^{a}\end{array}$ & $\begin{array}{l}60.50 \pm \\
1.15^{\mathrm{a}}\end{array}$ & $\begin{array}{l}59.10 \\
\pm 1.20^{\mathrm{a}}\end{array}$ & $\begin{array}{l}59.53 \pm \\
0.65^{a}\end{array}$ & $\begin{array}{l}60.17 \pm \\
0.80^{a}\end{array}$ \\
\hline $\begin{array}{l}\mathrm{MCH} \\
(\mathrm{pg})\end{array}$ & $\begin{array}{l}19.87 \pm \\
0.31^{\mathrm{a}}\end{array}$ & $\begin{array}{l}20.30 \pm \\
0.72^{a}\end{array}$ & $\begin{array}{l}19.90 \pm \\
0.61^{\mathrm{a}}\end{array}$ & $\begin{array}{l}19.83 \pm \\
0.35^{a}\end{array}$ & $\begin{array}{l}18.70 \pm \\
0.52^{a}\end{array}$ & $\begin{array}{l}19.53 \\
\pm 0.75^{a}\end{array}$ & $\begin{array}{l}19.20 \pm \\
0.53^{\mathrm{a}}\end{array}$ & $\begin{array}{l}19.92 \pm \\
0.78^{\mathrm{a}}\end{array}$ \\
\hline $\begin{array}{l}\mathrm{MCHC} \\
(\mathrm{g} / \mathrm{dL})\end{array}$ & $\begin{array}{l}33.63 \pm \\
1.14^{\mathrm{a}}\end{array}$ & $\begin{array}{l}33.10 \pm \\
0.50^{a}\end{array}$ & $\begin{array}{l}34.43 \pm \\
0.85^{a}\end{array}$ & $\begin{array}{l}33.53 \pm \\
0.45^{a}\end{array}$ & $\begin{array}{l}31.63 \pm \\
1.12^{a}\end{array}$ & $\begin{array}{l}32.53 \\
\pm 1.07 \\
a\end{array}$ & $\begin{array}{l}31.90 \pm \\
0.70^{a}\end{array}$ & $\begin{array}{l}33.70 \pm \\
1.00^{a}\end{array}$ \\
\hline $\begin{array}{l}\text { RDWCV } \\
(\%)\end{array}$ & $\begin{array}{l}15.87 \pm \\
0.21^{\mathrm{a}}\end{array}$ & $\begin{array}{l}16.97 \pm \\
1.53^{a}\end{array}$ & $\begin{array}{l}16.60 \pm \\
0.61^{\mathrm{a}}\end{array}$ & $\begin{array}{l}16.83 \pm \\
0.93^{a}\end{array}$ & $\begin{array}{l}16.47 \pm \\
0.32^{\mathrm{a}}\end{array}$ & $\begin{array}{l}16.53 \\
\pm 0.15^{a}\end{array}$ & $\begin{array}{l}15.90 \pm \\
0.56^{\mathrm{a}}\end{array}$ & $\begin{array}{l}17.23 \pm \\
1.50^{\mathrm{a}}\end{array}$ \\
\hline $\begin{array}{l}\text { RDWSCD } \\
\text { (fL) }\end{array}$ & $\begin{array}{l}36.53 \pm \\
1.64^{\mathrm{a}}\end{array}$ & $\begin{array}{l}41.3^{ \pm} \\
2.18^{b}\end{array}$ & $\begin{array}{l}38.43 \pm \\
0.55^{a}\end{array}$ & $\begin{array}{l}41.17 \pm \\
1.50^{\mathrm{b}}\end{array}$ & $\begin{array}{l}40.10 \pm \\
0.36^{a}\end{array}$ & $\begin{array}{l}39.50 \\
\pm 1.37\end{array}$ & $\begin{array}{l}37.87 \pm \\
1.76^{a}\end{array}$ & $\begin{array}{l}40.73 \pm \\
4.62^{a}\end{array}$ \\
\hline
\end{tabular}

Data are expressed as mean $\pm S D, n=4$. Values in the test groups carrying the same letter as the control group in the same-sex and in the same row are not significantly different according to Waller Duncan's Multiple Comparison Test $(p<0.05)$. WBCs: White blood cells, RBCs: Red blood cells, HCT: Hematocrit, PLT: Platelets, HGB: Haemoglobin, MCH: Mean corpuscular haemoglobin, MCHC: Mean corpuscular haemoglobin concentration, MCV: Mean corpuscular volume, Gran: Granulocytes, Lym: Lymphocytes, MO: Monocytes, RDWCV: Red blood cells distribution width CV, RDWSD: Red blood cells distribution width SD, PCT: Plateletcrit, MPV: mean platelet volume, PDW: Platelet distribution width. 


\begin{tabular}{|c|c|c|c|c|c|c|c|c|}
\hline \multirow{2}{*}{$\begin{array}{l}\text { Sexe } \\
\text { Dose } \\
(\mathrm{mg} / \mathrm{kg})\end{array}$} & \multicolumn{4}{|l|}{ Female } & \multicolumn{4}{|l|}{ Male } \\
\hline & 0 & 250 & 500 & 1000 & 0 & 250 & 500 & 1000 \\
\hline $\begin{array}{l}\text { PLT } \\
\left(10^{3} / \mu \mathrm{L}\right)\end{array}$ & $\begin{array}{l}888.67 \\
\pm \\
21.46^{b}\end{array}$ & $\begin{array}{l}730.33 \\
\pm \\
32.15^{a}\end{array}$ & $\begin{array}{l}717.33 \\
\pm \\
25.74^{\mathrm{a}}\end{array}$ & $\begin{array}{l}727.33 \\
\pm \\
44.77^{a}\end{array}$ & $\begin{array}{l}666.33 \\
\pm \\
29.16^{b}\end{array}$ & $\begin{array}{l}609 \pm \\
41.8^{\mathrm{ab}}\end{array}$ & $\begin{array}{l}597.67 \\
\pm \\
24.38^{a}\end{array}$ & $\begin{array}{l}569.67 \\
\pm 19.14^{\mathrm{a}}\end{array}$ \\
\hline РCT (\%) & $\begin{array}{l}0.61 \pm \\
0.02^{b}\end{array}$ & $\begin{array}{l}0.46 \pm \\
0.06^{\mathrm{a}}\end{array}$ & $\begin{array}{l}0.49 \pm \\
0.02^{\mathrm{a}}\end{array}$ & $\begin{array}{l}0.50 \pm \\
0.03^{\mathrm{a}}\end{array}$ & $\begin{array}{l}0.49 \pm \\
0.03^{b}\end{array}$ & $\begin{array}{l}0.43 \pm \\
0.05^{\mathrm{ab}}\end{array}$ & $\begin{array}{l}0.46 \pm \\
0.02^{b}\end{array}$ & $\begin{array}{l}0.37 \pm \\
0.02 \mathrm{a}\end{array}$ \\
\hline PDW (fL) & $\begin{array}{l}17.57 \pm \\
0.23^{a}\end{array}$ & $\begin{array}{l}17.33 \pm \\
0.55^{a}\end{array}$ & $\begin{array}{l}17.00 \pm \\
0.53^{\mathrm{a}}\end{array}$ & $\begin{array}{l}17.10 \pm \\
0.75^{a}\end{array}$ & $\begin{array}{l}17.03 \pm \\
0.47^{a b}\end{array}$ & $\begin{array}{l}18 \pm \\
0.50^{\mathrm{b}}\end{array}$ & $\begin{array}{l}16.73 \pm \\
0.29^{a}\end{array}$ & $\begin{array}{l}17.63 \pm \\
0.29^{b}\end{array}$ \\
\hline MPV (fL) & $\begin{array}{l}6.77 \pm \\
0.12^{\mathrm{a}}\end{array}$ & $\begin{array}{l}7.02 \pm \\
0.27^{a}\end{array}$ & $\begin{array}{l}6.83 \pm \\
0.12^{\mathrm{a}}\end{array}$ & $\begin{array}{l}6.83 \pm \\
0.12^{\mathrm{a}}\end{array}$ & $\begin{array}{l}6.90 \pm \\
00^{a}\end{array}$ & $\begin{array}{l}6.97 \pm \\
0.31^{a}\end{array}$ & $\begin{array}{l}6.60 \pm \\
0.17^{a}\end{array}$ & $\begin{array}{l}6.90 \pm \\
0.20^{a}\end{array}$ \\
\hline $\begin{array}{l}\text { Data are ex } \\
\text { control gro } \\
\text { Duncan's } \\
\text { Hematocri } \\
\text { corpuscula } \\
\text { Lymphocy } \\
\text { distributior }\end{array}$ & $\begin{array}{l}\text { pressed } \\
\text { up in the } \\
\text { ultiple C } \\
\text { PLT: Pla } \\
\text { haemoc } \\
\text { es, MO: N } \\
\text { width SL }\end{array}$ & $\begin{array}{l}\text { an } \pm \mathrm{SC} \\
\text {-sex an } \\
\text { rison T } \\
\text {, HGB: } \\
\text { concer } \\
\text { cytes, R } \\
\text { r: Platel }\end{array}$ & $\begin{array}{l}=4 . \text { Val } \\
\text { the sal } \\
\text { p }<0.0 \text { ! } \\
\text { moglok } \\
\text { ion, Mc } \\
\text { cV: Rec } \\
\text { it, MPV }\end{array}$ & $\begin{array}{l}\text { sin the t } \\
\text { row are } \\
\text { WBCs: } \\
\text { MCH: N } \\
\text { Mean c } \\
\text { ood cells } \\
\text { tean plat }\end{array}$ & $\begin{array}{l}\text { blood ce } \\
\text { corpuscl } \\
\text { cular vo } \\
\text { ribution } \\
\text { jolume, }\end{array}$ & $\begin{array}{l}\text { RBCs: } \\
\text { haem } \\
\text { ne, Gra } \\
\text { th CV, } \\
\text { W: Plat }\end{array}$ & $\begin{array}{l}\text { me letter } \\
\text { accordin } \\
\text { d blood } \\
\text { obin, Mc } \\
\text { iranuloc } \\
\text { WSD: Re } \\
\text { t distribu }\end{array}$ & $\begin{array}{l}\text { the } \\
\text { o Waller } \\
\text { s, HCT: } \\
\text { : Mean } \\
\text { s, Lym: } \\
\text { lood cells } \\
\text { n width. }\end{array}$ \\
\hline
\end{tabular}

Effect of TTF on urinary biochemical parameters. The results of the urinary and serum biochemical parameters of rats treated with methanol extract of TTF are presented in Table 4 . The extract did not affect urinary protein levels in either sex. There was a significant decrease $(p<0.05)$ in urinary creatinine from the $500 \mathrm{mg} / \mathrm{kg}$ dose onwards in female rats whereas no significant difference was observed in males compared to the control group. In both sexes, there was a significant increase $(p<0.05)$ in urine urea at $1000 \mathrm{mg} / \mathrm{kg}$ in females and $500 \mathrm{mg} / \mathrm{kg}$ in males compared to the control groups.

Table 4

Urinary biochemical parameters in males and females after 28 days of administration

\begin{tabular}{|c|c|c|c|c|c|c|c|c|}
\hline \multirow{2}{*}{$\begin{array}{l}\text { Sex } \\
\text { Doses } \\
(\mathrm{mg} / \mathrm{Kg})\end{array}$} & \multicolumn{4}{|l|}{ Female } & \multicolumn{4}{|l|}{ Male } \\
\hline & 0 & 250 & 500 & 1000 & 0 & 250 & 500 & 1000 \\
\hline $\begin{array}{l}\text { Creatinine } \\
(\mathrm{mg} / \mathrm{dL})\end{array}$ & $\begin{array}{l}2.85 \pm \\
0.13^{b}\end{array}$ & $\begin{array}{l}2.62 \pm \\
0.16^{\mathrm{b}}\end{array}$ & $\begin{array}{l}2.04 \pm \\
0.06^{\mathrm{a}}\end{array}$ & $\begin{array}{l}2.04 \pm \\
0.06^{\mathrm{a}}\end{array}$ & $\begin{array}{l}4.11 \pm \\
0.04^{b}\end{array}$ & $\begin{array}{l}3.86 \pm \\
0.05^{a}\end{array}$ & $\begin{array}{l}3.68 \pm \\
0.04^{a}\end{array}$ & $\begin{array}{l}3.49 \pm \\
0.06^{\mathrm{a}}\end{array}$ \\
\hline $\begin{array}{l}\text { Urea } \\
(\mathrm{mg} / \mathrm{dL})\end{array}$ & $\begin{array}{l}107.23 \\
\pm 4.26^{\mathrm{a}}\end{array}$ & $\begin{array}{l}104.07 \\
\pm 3.66^{\mathrm{a}}\end{array}$ & $\begin{array}{l}108.43 \\
\pm 2.25^{a}\end{array}$ & $\begin{array}{l}130.12 \\
\pm 2.51^{\mathrm{b}}\end{array}$ & $\begin{array}{l}106.93 \\
\pm 2.67^{a}\end{array}$ & $\begin{array}{l}113.41 \\
\pm 4.03^{a}\end{array}$ & $\begin{array}{l}131.93 \\
\pm 4.69^{b}\end{array}$ & $\begin{array}{r}133.73 \\
\pm 1.77^{6}\end{array}$ \\
\hline $\begin{array}{l}\text { Total } \\
\text { Protein } \\
\text { (g/dL) }\end{array}$ & $\begin{array}{l}0.79 \pm \\
0.05^{\mathrm{a}}\end{array}$ & $\begin{array}{l}0.79 \pm \\
0.05^{\mathrm{a}}\end{array}$ & $\begin{array}{l}0.85 \pm \\
0.05^{a}\end{array}$ & $\begin{array}{l}0.83 \pm \\
0.06^{\mathrm{a}}\end{array}$ & $\begin{array}{l}0.82 \pm \\
0.03^{a}\end{array}$ & $\begin{array}{l}0.86 \pm \\
0.06^{\mathrm{a}}\end{array}$ & $\begin{array}{l}0.85 \pm \\
0.05^{a}\end{array}$ & $\begin{array}{l}0.80 \pm \\
0.06^{a}\end{array}$ \\
\hline $\begin{array}{l}\text { Data are ex } \\
\text { control gro } \\
\text { Waller Dun }\end{array}$ & $\begin{array}{l}\text { ssed as } \\
\text { n the sa } \\
\text { 's Multip }\end{array}$ & $\begin{array}{l}\text { an } \pm \text { SD } \\
\text { gender } \\
\text { Compari }\end{array}$ & $\begin{array}{l}=4 \text {. Vall } \\
\text { in the } \\
\text { Test ( }\end{array}$ & $\begin{array}{l}\text { in the t } \\
\text { e row } \\
.05)\end{array}$ & oups & ng the & ne lette & $\begin{array}{l}\text { the } \\
\text { g to }\end{array}$ \\
\hline
\end{tabular}


Effect of TTF on serum biochemical parameters. Table 5 shows the results of serum biochemical parameters of the animals in the control groups and those that received different TTF doses $(250,500$, and $1000 \mathrm{mg} / \mathrm{kg} \mathrm{BW}$ ). In both male and female rats, after prolonged administration, a significant decrease $(p<0.05)$ in the values of transaminase activity (ALAT and ASAT), alkaline phosphatase, and total protein concentration were observed for all doses administered in comparison with the control group. In addition, a significant increase $(p<0.05)$ in urea levels was observed in male rats at all doses tested but was only observed in female rats at doses above $1000 \mathrm{mg} / \mathrm{kg}$. A significant decrease in serum creatinine was observed in male rats at all doses tested compared to the control group $(p<0.05)$.

Table 5

Serum biochemical parameters in males and females after 28 days of administration

\begin{tabular}{|c|c|c|c|c|c|c|c|c|}
\hline \multirow{2}{*}{$\begin{array}{l}\text { Doses } \\
(\mathrm{mg} / \mathrm{Kg})\end{array}$} & \multicolumn{4}{|l|}{ Female } & \multicolumn{4}{|l|}{ Male } \\
\hline & 0 & 250 & 500 & 1000 & 0 & 250 & 500 & 1000 \\
\hline $\begin{array}{l}\text { Creatinine } \\
(\mathrm{mg} / \mathrm{dL})\end{array}$ & $\begin{array}{l}0.78 \pm \\
0.02^{\mathrm{a}}\end{array}$ & $\begin{array}{l}0.78 \pm \\
0.05^{a}\end{array}$ & $\begin{array}{l}0.85 \pm \\
0.05^{a}\end{array}$ & $\begin{array}{l}0.84 \pm \\
0.03^{\mathrm{a}}\end{array}$ & $\begin{array}{l}1.44 \pm \\
0.03^{c}\end{array}$ & $\begin{array}{l}1.33 \pm \\
0.02^{b}\end{array}$ & $\begin{array}{l}1.27 \pm \\
0.01^{a}\end{array}$ & $\begin{array}{l}1.28 \pm \\
0.02^{\mathrm{a}}\end{array}$ \\
\hline $\begin{array}{l}\text { Urea } \\
(\mathrm{mg} / \mathrm{dL})\end{array}$ & $\begin{array}{l}71.23 \pm \\
3.35^{\mathrm{a}}\end{array}$ & $\begin{array}{l}71.23 \pm \\
1.58^{a}\end{array}$ & $\begin{array}{l}73.80 \pm \\
7.27^{a}\end{array}$ & $\begin{array}{l}87.35 \pm \\
2.56^{\mathrm{b}}\end{array}$ & $\begin{array}{l}62.35 \pm \\
0.78^{a}\end{array}$ & $\begin{array}{l}70.48 \pm \\
0.98^{b}\end{array}$ & $\begin{array}{l}72.29 \pm \\
2.25^{b c}\end{array}$ & $\begin{array}{l}74.25 \pm \\
1.86^{c}\end{array}$ \\
\hline $\begin{array}{l}\text { ASAT } \\
(\mathrm{U} / \mathrm{L})\end{array}$ & $\begin{array}{l}107.18 \\
\pm 2.31^{\mathrm{b}}\end{array}$ & $\begin{array}{r}106.96 \\
\pm 1.49^{b}\end{array}$ & $\begin{array}{l}94.62 \pm \\
2.57^{a}\end{array}$ & $\begin{array}{l}97.56 \pm \\
1.82^{\mathrm{a}}\end{array}$ & $\begin{array}{l}105.43 \\
\pm 2.31^{c}\end{array}$ & $\begin{array}{l}84.43 \pm \\
3.23^{\mathrm{b}}\end{array}$ & $\begin{array}{l}73.71 \pm \\
2.61^{\mathrm{a}}\end{array}$ & $\begin{array}{l}73.06 \pm \\
1.12^{\mathrm{a}}\end{array}$ \\
\hline $\begin{array}{l}\text { ALAT } \\
(\mathrm{U} / \mathrm{L})\end{array}$ & $\begin{array}{l}61.19 \pm \\
1.82^{\mathrm{b}}\end{array}$ & $\begin{array}{l}57.31 \pm \\
1.13^{\mathrm{a}}\end{array}$ & $\begin{array}{l}57.75 \pm \\
1.43^{a}\end{array}$ & $\begin{array}{l}57.09 \pm \\
1.31^{\mathrm{a}}\end{array}$ & $\begin{array}{l}65.19 \pm \\
2.08^{c}\end{array}$ & $\begin{array}{l}57.53 \pm \\
1.66^{\mathrm{b}}\end{array}$ & $\begin{array}{l}53.81 \pm \\
2.53^{\mathrm{a}}\end{array}$ & $\begin{array}{l}54.03 \pm \\
1.49^{a}\end{array}$ \\
\hline PAL (U/L) & $\begin{array}{l}363.43 \\
\pm 3.12^{d}\end{array}$ & $\begin{array}{l}308.26 \\
\pm 1.49^{c}\end{array}$ & $\begin{array}{l}261.29 \\
\pm 3.12^{b}\end{array}$ & $\begin{array}{l}200.18 \\
\pm 1.75^{\mathrm{a}}\end{array}$ & $\begin{array}{l}420.43 \\
\pm 2.35^{c}\end{array}$ & $\begin{array}{l}376.66 \\
\pm 2.35^{b}\end{array}$ & $\begin{array}{l}373.46 \\
\pm 3.12^{b}\end{array}$ & $\begin{array}{l}352.94 \\
\pm 2.35^{\mathrm{a}}\end{array}$ \\
\hline $\begin{array}{l}\text { Total } \\
\text { Protein } \\
\text { (g/dL) }\end{array}$ & $\begin{array}{l}8.70 \pm \\
0.29^{b}\end{array}$ & $\begin{array}{l}7.66 \pm \\
0.22^{\mathrm{a}}\end{array}$ & $\begin{array}{l}7.37 \pm \\
0.58^{\mathrm{a}}\end{array}$ & $\begin{array}{l}7.02 \pm \\
0.42^{\mathrm{a}}\end{array}$ & $\begin{array}{l}10.31 \pm \\
0.07^{b}\end{array}$ & $\begin{array}{l}9.13 \pm \\
0.85^{\mathrm{a}}\end{array}$ & $\begin{array}{l}8.38 \pm \\
0.70^{\mathrm{a}}\end{array}$ & $\begin{array}{l}7.92 \pm \\
0.59^{\mathrm{a}}\end{array}$ \\
\hline $\begin{array}{l}\text { Data are ex } \\
\text { control gro } \\
\text { Waller Dun } \\
\text { Alanine an }\end{array}$ & 's Multi & $\begin{array}{l}\text { gender } \\
\text { Compar } \\
\text { e, PAL: }\end{array}$ & sphatas & $\begin{array}{l}\text { (05). AS } \\
\text { kaline }\end{array}$ & spă & $\begin{array}{l}\text { ig th } \\
\text { tly di } \\
\text { nino }\end{array}$ & $\begin{array}{l}\text { lette } \\
\text { acc } \\
\text { eras }\end{array}$ & $\begin{array}{l}\text { the } \\
\text { g to } \\
\text { AT: }\end{array}$ \\
\hline
\end{tabular}

Effect of TTF on lipid profile of male and female rats after 28 days of oral treatment. All the lipid profile values obtained from the serum of the control and test groups at different doses $(250,500$, and 1000 $\mathrm{mg} / \mathrm{kg})$ after 28 days of oral administration are presented in Table 6. A significant decrease $(p<0.05)$ in triacylglycerol (TAG) was observed in female rats from the dose of $500 \mathrm{mg} / \mathrm{kg}$. LDL cholesterol varied significantly, by decreasing $(p<0.05)$ at all doses tested in both males and females compared to the control group. A significant increase $(p<0.05)$ in total cholesterol and HDL cholesterol values was observed in both males and females compared to the control group for all different doses tested. 
Table 6

Lipid profile parameters in females and males after 28 days of administration

\begin{tabular}{|c|c|c|c|c|c|c|c|c|}
\hline \multirow{2}{*}{$\begin{array}{l}\text { Doses } \\
(\mathrm{mg} / \mathrm{Kg})\end{array}$} & \multicolumn{4}{|l|}{ Female } & \multicolumn{4}{|l|}{ Male } \\
\hline & 0 & 250 & 500 & 1000 & 0 & 250 & 500 & 1000 \\
\hline $\begin{array}{l}\text { TAG } \\
(\mathrm{mg} / \mathrm{kg})\end{array}$ & $\begin{array}{l}62.45 \pm \\
2.25^{b}\end{array}$ & $\begin{array}{l}62.83 \pm \\
1.29^{b}\end{array}$ & $\begin{array}{l}57.23 \\
\pm 3.09^{a}\end{array}$ & $\begin{array}{l}50.94 \pm \\
3.96^{\mathrm{a}}\end{array}$ & $\begin{array}{l}67.17 \pm \\
3.08^{a}\end{array}$ & $\begin{array}{l}71.13 \pm \\
3.28^{a}\end{array}$ & $\begin{array}{l}69.81 \pm \\
2.35^{a}\end{array}$ & $\begin{array}{l}65.85 \pm \\
3.04^{\mathrm{a}}\end{array}$ \\
\hline $\begin{array}{l}\mathrm{T}-\mathrm{CHOL} \\
(\mathrm{mg} / \mathrm{Kg})\end{array}$ & $\begin{array}{l}82.60 \pm \\
1.77^{a}\end{array}$ & $\begin{array}{l}90.54 \pm \\
1.57^{b}\end{array}$ & $\begin{array}{l}95.96 \\
\pm 2.89^{c}\end{array}$ & $\begin{array}{l}103.78 \\
\pm 3.2^{d}\end{array}$ & $\begin{array}{l}88.78 \pm \\
1.30^{\mathrm{a}}\end{array}$ & $\begin{array}{l}93.82 \pm \\
3.80^{\mathrm{ab}}\end{array}$ & $\begin{array}{l}97.23 \pm \\
4.59^{b}\end{array}$ & $\begin{array}{l}112.86 \\
\pm 2.58^{\mathrm{C}}\end{array}$ \\
\hline $\begin{array}{l}\mathrm{HDL} \\
(\mathrm{mg} / \mathrm{Kg})\end{array}$ & $\begin{array}{l}52.71 \pm \\
2.12^{a}\end{array}$ & $\begin{array}{l}62.04 \pm \\
0.71^{b}\end{array}$ & $\begin{array}{l}68.98 \\
\pm 3.01^{c}\end{array}$ & $\begin{array}{l}81.34 \pm \\
3.17^{d}\end{array}$ & $\begin{array}{l}53.68 \pm \\
1.94^{\mathrm{a}}\end{array}$ & $\begin{array}{l}66.33 \pm \\
2.78^{b}\end{array}$ & $\begin{array}{l}69.99 \pm \\
4.84^{b}\end{array}$ & $\begin{array}{l}83.23 \pm \\
2.97^{c}\end{array}$ \\
\hline $\begin{array}{l}\text { LDL } \\
(\mathrm{mg} / \mathrm{Kg})\end{array}$ & $\begin{array}{l}18.70 \pm \\
1.14^{c}\end{array}$ & $\begin{array}{l}15.93 \pm \\
1.30^{\mathrm{b}}\end{array}$ & $\begin{array}{l}15.51 \\
\pm 1.38^{\mathrm{b}}\end{array}$ & $\begin{array}{l}12.26 \pm \\
1.03^{\mathrm{a}}\end{array}$ & $\begin{array}{l}21.66 \pm \\
0.84^{b}\end{array}$ & $\begin{array}{l}13.26 \pm \\
2.27^{a}\end{array}$ & $\begin{array}{l}13.28 \pm \\
1.28^{\mathrm{a}}\end{array}$ & $\begin{array}{l}16.46 \pm \\
2.78^{a}\end{array}$ \\
\hline
\end{tabular}

Histological sections of the liver and kidneys. Histopathological examinations were performed on the liver and the kidney to assess organ damage. The kidney of treated rats showed normal glomeruli and there was no necrosis of tubular epithelium either in female (Fig. 4) or male (Fig. 5) treated rats. Adverse effects were neither found in the liver of female (Fig. 6) nor male (Fig. 7) treated rats compared to the control group.

\section{Discussion}

According to the new Globocan estimates, the global cancer burden has increased to 19.3 million cases and 10 million cancer deaths in 2020 [1]. Cancer treatments including chemotherapy, immunotherapy, and hormone therapy have significantly improved patient survival in recent years. Although these drugs have a significant impact on reducing the mortality of cancer patients, they have numerous side effects including nephrotoxicity, hepatotoxicity, and cardiotoxicity, etc. [42-43]. This toxicity forces a reduction of the doses leading inevitably to the emergence of multidrug resistance against conventional anticancer drugs. Therefore, the challenge in the fight against cancer is to find anticancer drugs with acceptable toxicity, and capable not only of eliminating sensitive cancers but above all of defeating those with resistant and metastatic phenotypes. In the present work, the crude extract of TTF presented a 100\% spectrum of activity on human and animal metastatic colorectal and melanoma cancer cell lines with significant $\mathrm{IC}_{50}$ values below $20 \mu \mathrm{g} / \mathrm{mL}$ on more than $50 \%$ of the tested cell lines [2]. This result is justified by its composition in secondary metabolites rich in molecules with proven anticancer activity as demonstrated in a previous study [44]. Similarly, oleanolic acid $\mathrm{N}$-acetylglycoside which is a compound previously isolated from the dichloromethane methanol extract of the fruits of this plant exhibited a $100 \%$ antiproliferative activity spectrum on a panel of 18 resistant and metastatic cancer cell lines with $\mathrm{IC}_{50}$ 
values ranging from $3.18 \mu \mathrm{M}$ (CCRF-CEM leukemia cells) to $9.56 \mu \mathrm{M}$ (HepG2 hepatocarcinoma cells) [45]. In an earlier reported study, TTF induced apoptosis in CCRF-CEM cells, mediated by the alteration of the mitochondrial membrane potential (MMP) and increased in the production of reactive oxygen species (ROS) in CCRF-CEM cells, whilst one of its constituents, olean-12-en-3- $\beta-O_{-D}$-glucopyranoside also induced apoptosis mediated by caspases activation, MMP alteration and increased ROS production [44]. TTF was also cytotoxic towards MCF-7 cells and Jurkat cells with $\mathrm{IC}_{50}$ values of $380.12 \mu \mathrm{g} / \mathrm{mL}$ and $340.47 \mu \mathrm{g} / \mathrm{mL}$, respectively [22]. These data are weaker than those obtained in the present work, probably showing the dichlomethane-methanol might be the best extractive solvent of the cytotoxic components of the plant. Herein, the recorded activity spectra were $100 \%$ for compound $\mathbf{5}, 88.88 \%$ for compound $\mathbf{2}, \mathbf{7 7 . 7 7 \%}$ for compound 6 , and $66.66 \%$ for compound 7 . These results are an indication that these phytochemicals are potential anticancer agents. Regarding the interesting antiproliferative activity of the extract and its isolates, further toxicity studies are planned in an animal model.

Although plants are traditionally used in the management of several diseases, their toxicity is not excluded. Many previous works related to the study of the toxicity of plant extracts demonstrated their aggressiveness towards many organs altering their physiological functions [45-46]. It has also been reported that repeated and prolonged administration of methanol extract of some spices such as Piper capenses and Imperata cylindrica at doses higher than $500 \mathrm{mg} / \mathrm{kg}$ BW provoked adverse effects on liver and kidney function [47-48]. In the present study, the toxicity of dichloromethane methanol extract of TTF after its acute and sub-chronic administration was evaluated in Wistar strain albino rats. The results in the acute study indicated that oral administration of TTF at a single dose of $5000 \mathrm{mg} / \mathrm{kg} \mathrm{did} \mathrm{not}$ induce any mortality and no signs of toxicity in rats. This suggested an $L_{50}$ value greater than 5000 $\mathrm{mg} / \mathrm{kg}$ indicating that TTF is practically non-toxic [49]. Moreover, according to the OECD (2008a), orally ingested substances with an $L D_{50}$ value $>5000 \mathrm{mg} / \mathrm{kg}$ are considered relatively safe. This result corroborates those of many other studies which also demonstrated that the $\mathrm{LD}_{50}$ following a single dose of the extracts of certain spices was higher than $5000 \mathrm{mg} / \mathrm{kg} \mathrm{BW}[47,48]$. The work of Dongmo et al. (2019) [24]. on the aqueous extract of the bark of T. tetraptera also led to a similar result. On the other hand, the study of the acute toxicity of the ethanolic extract of the fruits of this plant on a fish culture showed a mortality of $50 \%$ after $54 \mathrm{~h}$ of exposure to a concentration of $45 \mathrm{mg} / \mathrm{L}$ of extract [50]. The difference observed compared to our study could be justified by the difference in the extraction solvent used and the exposure time which was relatively shorter in our study where the animals received a single dose by gavage.

Repeated administration over a period of 28 days did not cause mortality or signs of systemic toxicity in the test animals. After analysis of the results, no significant difference was observed in the relative organ weights of the treated animals compared to the control group. This would be justified by the absence of compounds in the dichloromethane methanol extract of TTF that cause excessive organ growth. It is important to note that some plant extracts can have hypertrophic effects on organs exposing the organism to develop cancers [51]. Daily administration for a period of 28 days of TTF showed a significant increase in white blood cells. 
The hematopoietic system is known to be highly sensitive to toxic substances and is also a significant indicator of physiological and pathological conditions in humans and animals [52]. In this study, the dichloromethane methanol extract of TTF significantly $(p<0.05)$ increased the levels of white blood cells (WBC) in females receiving the highest dose compared to the control group. This could be attributed to the boosting capacity ofthe immune system of the test animals by the bioactive molecules in the extract [53]. This is not in agreement with other works that have shown a decrease in the level of white blood cells following the treatment of animals with ethanolic extract of the fruits of this plant [54]. This could be explained by the difference in the extraction solvent which was dichloromethane methanol in the present work. The non-significant effect of the extract on the RBC may be an indication that the balance between the rate of production (erythropoiesis) and destruction of the blood corpuscles was not altered. Therefore, the significant decrease $(p<0.05)$ in HGB, PCT, and MCH levels in females treated with the highest dose could mean that the incorporation of hemoglobin into the red blood cells and the morphology of the red blood cells were altered [55]. The platelet counts and platelet blood volume (PCT) also decreased $(p<0.05)$ appreciably at all treated dosages of the extract in both females and males. This could be explained by the ability of the extract to prevent the development and fragmentation of megakaryocytes in the bone marrow. The liver plays a major role in the detoxification and excretion of many endogenous and exogenous compounds; any damage or impairment of its functions can have many consequences for human and animal health [56]. Liver damage is associated with cellular necrosis, increased tissue lipid peroxidation, and depletion of reduced glutathione levels. In addition, serum levels of many biochemical markers such as transaminases, alkaline phosphatase, triglycerides, and cholesterol are elevated in liver disease [56]. The evaluation of liver function in this study through serum biochemical parameters such as ALT, ASAT, PAL, and total protein showed a significant decrease $(p<$ 0.05 ) in all these parameters and at all doses tested. Based on these results, TTF would not affect the proper functioning of the liver because any hepatocellular injury would have resulted in a serum increase in ALT and AST levels [57]. This could be justified by a hepatoprotective effect of the extract due to its phenolic compounds which play an antioxidant role in preventing the peroxidation of membrane lipids [58-59].

The primary function of the kidneys is to eliminate the toxic waste produced by the normal functioning of the body and transported by the blood. Indeed, many previous works strongly confirm the ability of plant extracts to act in the kidney as powerful scavengers of free radicals; preventing their toxic effects on lipid peroxidation responsible for the upward variation of biochemical parameters such as creatinine and urea via the destruction of membranes [60-61]. This study jointly showed a significant decrease $(p<0.05)$ in urinary and serum creatinine in male rats at all doses, and from dose $500 \mathrm{mg} / \mathrm{kg}$ in females for serum creatinine. This could be justified by the nephroprotective effect of TTF extract through the antioxidant capacity of some of its constituents such as flavonoids and total phenols which would prevent membrane lysis [62]. On the contrary, a significant increase $(p<0.05)$ in urinary and serum urea was found in male rats from $500 \mathrm{mg} / \mathrm{kg}$ onwards and at all doses tested. This could notify a toxic effect of the extract from the dose $500 \mathrm{mg} / \mathrm{kg}$. This result is in contradiction with that of other works confirming the protective effect of methanol extract of TTF on kidney cells by inhibition of xanthine oxidase 
responsible for lipid peroxidation and membrane destabilization [59]. The lipid profile is a set of parameters among which LDL-cholesterol, total cholesterol, and triglycerides are those that are mainly involved in the risk factors of cardiovascular diseases [63]. The significant decrease $(p<0.05)$ in triacylglycerol, LDL as well as the increase in HDL and total cholesterol levels could be explained by the ability of the different components of TTF extract to improve the catabolism of cholesterol into bile acids [64]. Indeed, many previous works have demonstrated the lipid-lowering and anti-obesity potential of $T$. tetraptera fruit extracts $[23,65]$.

\section{Conclusion}

This work aimed to demonstrate the antiproliferative activity towards human and animal cell lines of the dichloromethane methanol extract of TTF and its isolated compounds and to evaluate the toxicological profile of the extract on albino rats of Wistarstrain. The crude extract and the tested compounds, especially $\mathbf{5}$ showed cytotoxic activity towards human and animal carcinoma cell lines. The acute toxicity results of TTF suggested that the dichloromethane-methanol extract of TTF is almost nontoxic.

Nonetheless, it should be taken with caution during a sub-chronic administration to avoid renal damages.

\section{Abbreviations}

ALAT, alanine aminotransferase; ALP, alkaline phosphatase; ASAT, aspartate aminotransferase; BW, body weight; DMSO, dimethylsulfoxide; EDTA, ethylenediaminetetraacetic acid; $\mathrm{Hb}$, hemoglobin; HCT, hematocrit; $H D L$, high-density lipoprotein; $L D_{50}, 50 \%$ lethal dose; $L D L$, low-density lipoprotein; $M C H$, mean corpuscular hemoglobin; $\mathrm{MCH}$, mean corpuscular hemoglobin; $\mathrm{MCHC}$, mean corpuscular hemoglobin concentration; $\mathrm{MCHC}$, mean corpuscular hemoglobin concentration; $\mathrm{MCV}$, mean corpuscular volume; $\mathrm{MCV}$, mean corpuscular volume; OECD, Organisation for Economic Cooperation, and Development;PLT, platelets; RBC, red blood cells; RRA, resazurin reduction assay; TC, total cholesterol; TG, triglyceride; TTF, extract of $T$. tetraptera fruits.

\section{Declarations}

\section{Ethics approval and consent to participate}

All experiments were conducted in accordance with established guidelines for the use of animals in research.All sections of this report adhere to the ARRIVE Guidelines for reporting animal research. A completed ARRIVE guidelines checklist is included in Checklist S1.The research project was approved by the Faculty of Science of the University of Dschang. The plant was identified and authenticated by $\mathrm{Mr}$ NANA Victor as Tetrapleura tetraptera (Schumm. \&Thonn.) Taub in comparison with the specimen of the herbarium under the voucher number 19785 SRF/Cam.

\section{Consent for application}

Not applicable 
Availability of data and materials

All data generated or analysed during this study are included in this published article and its supplementary information files.

\section{Competing interests}

VK is a Section Editor of BMC Complementary and Alternative Medicine; ATM is an Associate Editor of BMC Complementary and Alternative Medicine.The remaining authors declare that they have no competing interests.

\section{Funding}

This study was financially supported by the Alexander von Humboldt Foundation

\section{Authors' Contribution}

INB, ATM, GSN, and GFC carried out the experiments. VK and TE designed the study. INB and ATM wrote the manuscript. VK, TE supervised the work, corrected the manuscript, and provided the facilities for the study. All authors read and approved the final manuscript.

\section{Acknowledgements}

ATM is thankful to Alexander von Humboldt Foundation for the 18 months fellowship in Prof. Dr. Thomas Efferth's laboratory in Mainz, Germany through the "Georg Foster Research Fellowship for Experienced Researcher" program.ATM andVK are also grateful to the Alexander von Humboldt Foundation for the 3 months further research stay fellowship at home from November 2021 to January 31, 2022.

\section{References}

1. Sung H, Ferlay J, Siegel RL, Laversanne M, Soerjomataram I, Jamal and Bray f. GlobalCancer Statistics 2020: GLOBOCAN Estimates of Incidence and Mortality Worldwide for 36 Cancers in 185 Countries. CA: A Cancer J. Clin. 2021; 71, 209-249.

2. Kuete V, Efferth T. African Flora Has the Potential toFight Multidrug Resistance of Cancer. BioMed Res. Int.2015, 914813.

3. Miller KD, Siegel RL, Lin CC, Mariotto AB. Cancer treatment and survivorship statistics. CA Cancer J. Clin.2016; 66, 271-289.

4. Mbaveng AT, Kuete V, and Efferth T.Potential of Central, Eastern and Western Africa Medicinal Plants for Cancer Therapy: Spotlight on Resistant Cells and Molecular Targets. Frontiers in Pharmacology. 2017; 8, 343.

5. Nayim P, Mbaveng AT, Sanjukta M, Rikesh J, Kuete V, Sudhir K. CD24 gene inhibition and TIMP-4 gene upregulation by Imperatacylindrica's root extract prevents metastasis of CaSki cells via 
inhibiting PI3K/Akt/snail signaling pathway and blocking EMT. J. Ethnopharmacol. 2021a; 275, 114111.

6. Nayim P, Sudhir K, Mbaveng TA, Kuete V, Sanjukta M. In vitro anticancer activity of Imperata cylindrica root's extract toward human cervical cancer and identification of potential bioactive compounds. Biomed Res. Int.2021b, 12.

7. Wamba BE N, Ghosh P, Mbaveng AT, Bhattacharya S, Debarpan M, Depanwita S, Saunak MM, Kuete $\mathrm{V}$, Nabendu M. Botanical from Piper capense fruit can help to combat the melanoma as demonstrated by in vitro and in vivo studies. Evid. Based Complement Alternat. Med. 2021; 8810368.

8. Fankam AG, Kuete V, Voukeng IK, Kuiate JR, Pages JM. Antibacterial activities of selected Cameroonian spices and their synergistic effects with antibiotics against multidrug-resistant phenotypes. BMC Complement. Altern. Med. 2011; 11, 104.

9. Kuete V, Krusche B, Youns M, Voukeng I, Fankam AG, Tankeo S, Lacmata S, Efferth T. Cytotoxicity of some Cameroonian spices and selected medicinal plant extracts. J. Ethnopharmacol. 2011; 134, 803-812.

10. Tekwu EM, Askun T, Kuete V, Nkengfack AE, Nyasse B, Etoa FX, Beng VP. Antibacterial activity of selected Cameroonian dietary spices ethno-medically used against strains of Mycobacterium tuberculosis. J. Ethnopharmacol. 2012; 142, 374-382.

11. Voukeng IK, Kuete V, Dzoyem JP, Fankam AG, Noumedem JA, Kuiate JR, Pages JM. Antibacterial and antibiotic-potentiation activities of the methanol extract of some Cameroonian spices against Gramnegative multi-drug resistant phenotypes. BMC Res. Notes. 2012; 5, 299.

12. Fankam AG, Kuiate JR, Kuete V. Antibacterial activities of Beilschmiedia obscura and six other Cameroonian medicinal plants against multi-drug resistant Gram-negative phenotypes. BMC Complement. Altern. Med. 2014; 14, 241.

13. Seukep JA, Fankam AG, Djeussi DE, Voukeng IK, Tankeo SB, Noumdem JA, Kuete AH, Kuete V. Antibacterial activities of the methanol extracts of seven Cameroonian dietary plants against bacteria expressing MDR phenotypes. Springer Plus. 2013; 2, 363.

14. Tamokou JDD, Mbaveng AT, Kuete V. Chapter 8 - Antimicrobial activities of African medicinal spices and vegetables. Medicinal Spices and Vegetables from Africa. Academic Press. 2017; pp. 207-237.

15. Nayim, P., Mbaveng, A.T., Wamba, B.E.N., Fankam, A.G., Dzotam, J.K., Kuete, V., 2018. Antibacterial and antibiotic-potentiating activities of thirteen Cameroonian edible plants against Gram-negative resistant phenotypes. ScientificWorldJournal 2018, 4020294.

16. Bachar SC, Mazumder K, Bachar R, Aktar A, Al Mahtab M. A review of medicinal plants with antiviral activity available in bangladesh and mechanistic insight into their bioactive metabolites on SARSCoV-2, HIV and HBV. Front. Pharmacol. (2021); 12, 732-891.

17. Adesina SK, Iwalewa EO, Johnny II. Tetrapleura tetraptera TaubEthnopharmacology, Chemistry, Medicinal and Nutritional Values.Br. J. Pharm. Res. 2016; 12, 1-22.

18. Epoh NJ, Dongmo OLM, Tchouanguep FM, Telefo PB. Ethnobotanical Study of Medicinal Plants used as AntiObesity Remedies in Foumban and Dschang Cities (West-Cameroon). Eur. J. Med. Plant. 
2020; 31, 54-70.

19. Igwe OU, Akabuike HC. Free radical scavenging activity, phytochemistry and antimicrobial properties of Tetrapleura tetraptera Seeds. Int. Res. J. Chem. Chem. Sci. 2016; 3, 037-042.

20. Ozaslan M, Karagoz ID, Lawal RA, Kilic IH, Cakir A, Odesanmi OS, Guler I. and Ebuehi OAT. Cytotoxic and anti-proliferative activities of the Tetrapleura tetraptera fruit extract on Ehrlich ascites tumor cells. Int. J. Pharmacol. 2016; 12, 655-662.

21. Mbaveng TA, Chi GF, BonsouNI, Ombito JO, Yeboah SO, Kuete V, Efferth T. Cytotoxic phytochemicals from the crude extract of Tetrapleura tetraptera fruits towards multi-factorial drug resistant cancer cells. J. Ethnopharmacol. 2021; 267, 113632.

22. Aikins AR, Birikorang PA, Chama M, Dotse E, Anning A, and Appiah-Opong R. Antiproliferative Activities of Methanolic Extract and Fractions of Tetrapleura Tetraptera Fruit.Evid. Based Complement. Alternat. Med. 2021; 4051555.

23. Kuate D, Kengne APN, Biapa CPN, Azantsa BGK, Wan Muda WAMB. Tetrapleura tetraptera spice attenuates high-carbohydrate, high-fat diet-induced obese and type 2 diabetic rats with metabolic syndrome features. Lipids in Health and Disease. 2015; 14, 1-13.

24. Dongmo MOL, Epoh NJ, Tadjoua TH, Yousuf S, Telefo PB, Tapondjou AL, Choudhary MI. Acute and sub-acute toxicity of the aqueous extract from the stem bark of TetrapleuratetrapteuraTaub. (Fabaceae) in mice and rats. J. Ethnopharmacol.2019; 236, 42-49.

25. Fogh J, Fogh JM, Orfeo T. One hundred and twenty-seven cultured human tumor cell lines producing tumors in nude mice. J. Natl. Cancer Inst. 1977; 59, 221-226.

26. Schadendorf D, Fichtner I, Makki A, Alijagic S, Kiipper M, Mrowietz U, Henz BM. Metastatic potential of human melanoma cells in nude mice characterisation of phenotype, cytokine secretion and tumour-associated antigens. Br. J. Cancer. 1996; 74: 194-199.

27. White SB, Procissi D, Chen J, Gogineni VR, Tyler P, Yang Y, Omary RA, Larson AC. Characterization of CC-531 as a rat model of colorectal liver metastases. PLoS One. 2016; 11, 155-334.

28. Mbaveng AT, Noulala CGT, Samba ARM, Tankeo SB, Abdelfatah S, Fotso GW, Happi EN, Ngadjui BT, Beng VP, Kuete V, EfferthT. The alkaloid, soyauxinium chloride, displays remarkable cytotoxic effects towards a panel of cancer cells, inducing apoptosis, ferroptosis and necroptosis. Chem. Biol. Interact. $2021 ; 333,109334$.

29. Mbaveng AT, Damen F, Guefack MF, Tankeo SB, Abdelfatah S, Bitchagno GTM, Çelik I, Kuete V, Efferth T. 8,8-bis-(Dihydroconiferyl)-diferulate displayed impressive cytotoxicity towards a panel of human and animal cancer cells. Phytomedicine2020; 70, 153215.

30. Mbaveng AT, Chi GF, Nguenang GS, Abdelfatah S, Tchangna Sop RV, Ngadjui BT, Kuete V, Efferth T. Cytotoxicity of a naturally occuring spirostanol saponin, progenin III, towards a broad range of cancer cell lines by induction of apoptosis, autophagy and necroptosis. Chem. Biol. Interact. 2020; 326, 109141.

31. O'Brien J, Wilson I, Orton T, Pognan F. Investigation of the Alamar Blue (resazurin) fluorescent dye for the assessment of mammalian cell cytotoxicity. Eur. J. Biochem. 2000; 267, 5421-5426. 
32. Kuete V, Nkuete AHL, Mbaveng AT, Wiench B, Wabo HK, Tane P, Efferth T. Cytotoxicity and modes of action of 4'-hydroxy-2',6'-dimethoxychalcone and other flavonoids toward drug-sensitive and multidrug-resistant cancer cell lines. Phytomedicine. 2014; 21, 1651-1657.

33. Kuete V, Mbaveng AT, Nono EC, Simo CC, Zeino M, Nkengfack AE, Efferth T. Cytotoxicity of seven naturally occurring phenolic compounds towards multi-factorial drug-resistant cancer cells. Phytomedicine. 2016; 23, 856-863.

34. Kuete V, Mbaveng AT, Sandjo LP, Zeino M, Efferth T. Cytotoxicity and mode of action of a naturally occurring naphthoquinone, 2-acetyl-7-methoxynaphtho[2,3-b]furan-4,9-quinone towards multifactorial drug-resistant cancer cells. Phytomedicine. 2017; 33, 62-68.

35. Adem FA, Mbaveng AT, Kuete V, Heydenreich M, Ndakala A, Irungu B, Yenesew A, Efferth T. Cytotoxicity of isoflavones and biflavonoids from Ormocarpumkirkiitowards multi-factorial drug resistant cancer. Phytomedicine. 2019b; 58, 152853

36. Mbaveng AT, Bitchagno GTM, Kuete V, Tane P, Efferth T. Cytotoxicity of ungeremine towards multifactorial drug resistant cancer cells and induction of apoptosis, ferroptosis, necroptosis and autophagy. Phytomedicine. 2019; 60, 152832.

37. Telefo B.P. “Contribution a` l'étude des plantesmédicinales du Cameroun: influence de l'extraitaqueux du mélange des feuillesd'AloebuettneriA. Berger (liliacées), Diclipteriaverticillata G.J.H. Amshoff (Acanthacées), Hibiscus macranthusHochstex. A rich (Malvacées), Justicia insularis T. Anders (Acanthacées) sur certains paramètres biochimiques et physiologiques de la reproduction chez les rattes," thèse de doctorat, 3ème cycle, Université de Yaoundé, Cameroun. (1998).

38. Kilkenny C, Browne WJ, Cuthill IC, Emerson M, Altman DG. Improving bioscience research reporting: the ARRIVE guidelines for reporting animal research. PLoS Biol. 2010;8:e1000412.

39. OECD. Guidelines for the testing of chemicals: Test № 425, Acute Oral Toxicity - Up-and-DownProcedure; 2008. p. 1-29.

40. Galigher AE, and Kozloff EN. Esentials of Practical Microtechniques. Lea and Febiger, Philadelphia, PA., USA. 1971.

41. Kamsu GT, Fodouop SPC, Tagne RS, Kodjio N, Fakam ALN, Gatsing D. Evaluation of the Acute and Sub-chronic Toxicity of the Ethanolic Extract of Curcuma longa (Zingiberaceae) in Wistar Albino Rats. Modern Chemistry \& Applications. $2019 ; 7:$ :267

42. Shakira, D.K., Rasul, K.I., 2009. Chemotherapy induced cardiomyopathy: pathogenesis, monitoring and management. J. Clin. Med. Res. 1, 8-12.

43. Brana I, Tabernero J. Cardiotoxicity. Ann. Oncol. 21 Suppl 7, vii 2010; 173-179.

44. Mbaveng, A.T., Chi, G.F., Bonsou, I.N., Abdelfatah, S., Tamfu, A.N., Yeboah, E.M.O., Kuete, V., Efferth, T., 2020. N-acetylglycoside of oleanolic acid (aridanin) displays promising cytotoxicity towards human and animal cancer cells, inducing apoptotic, ferroptotic and necroptotic cell death.Phytomedicine76, 153261.

45. Seremet OC, Olaru OT, Gutu CM, Nitulescu GM, Ilie M, Negres S, Zbarcea CE, Purdel CN, Spandidos DA, Tsatsakis AM, Coleman MD, Margina DM. Toxicity of plant extracts containing pyrrolizidine 
alkaloids using alternative invertebrate models. Mol. Med. Rep. 2018. 17: 7757-7763.

46. Muia BM, Mbaria JM, Kanja LW, Gitahi N, Okumu PO, Okumu MO. Acute and sub-acute toxicity study of the root extractsof Fagaropsishildebrandtiinn mice and evaluation of theirantimicrobial effects. F1000Research. 2020; 8, 1444.

47. Nayim P, Mbaveng AT, Ntyam AM, Kuete V. A botanical from the antiproliferative Cameroonian spice, Imperata cylindrica is safe at lower doses, as demonstrated by oral acute and sub-chronic toxicity screenings. BMC Complement. Med. Ther. 2020; 20, 273.

48. Wamba BEN, Mbaveng AT, Tazoho GM, Kuete V. Botanical from the medicinal spice, Piper capense is safe as demonstrated by oral acute and subchronic toxicity investigations. Heliyon. 2020; 6, e05470.

49. Loomis TA, Hayes AW. Loomis's essentials of toxicology. 4th ed., California, Academic press: (1996); 208-245.

50. Omitoyin BO, Ogunsanmi AO \& Adesina BT Studies on acute toxicity of piscicidal plant (Tetrapleura tetraptera) extract on tilapia (Sarotherodongalilaeus) fingerlings. Tropical Journal of Animal Science 1999; 2(2): 191-197

51. Maina VA, Garba A, Maurice NA, Baraya YS, Owada AH, Hambolu SE, Sada A, Agang I, Gashua MM, Sa'adatu I, Gugong VT. Effect of dose rates on organs weight in leptadeniahastataextract treated white albino rats.J. Exp. Biol. Agric. Sci. 2013; 1, 1.

52. Orong LX, Yongjiang L, Lijuan W, Yuhan LG, Xue SY, Cui Y, Xue M. Acute and subacute toxicity of ethanolic extracts from Salvia przewalskii Maxim in rodents. J. Ethnopharmacol. 2010; 131: 110-115

53. Idoh K, Agbonon A, Potchoo Y, Gbeassor M. Toxicological assessment of the hydroethanolic leaf extract of Clerodendrum capitatum in Wistar rats. Pan Afr. Med. J. 2016; 24, 66.

54. Odesanmi SO, Lawal RA, Ojokuku SA. Haematological effects of ethanolic fruit extract of Tetrapleura tetraptera in male Dutch white Rabbits. Res. J. Med. Plant. 2010. 4, 213-217.

55. Selmanoglu G, Barlas N, Songür S, Koçkaya EA. Carbendazim-induced haematological, biochemical and histopathological changes to the liver and kidney of male rats. Hum. Exp. Toxicol. 2001; 20, 625230.

56. Subramanian S, Khan HBH, Elumalai N, Lakshmi SYS. Hepatoprotective effect of ethanolic extract of whole plant of Andrographis paniculate against $\mathrm{CCl}_{4}$-induiced hepatotoxicity in rats. Comp. Clin. Path. 2015; 24, 1-7.

57. Meyer D, Harvey JW. Hepatobiliary and skeletal muscle enzymes and liver function tests. In: Meyer D, Harvey JW, editors. Veterinary laboratory medicine: interpretation and diagnosis. St. Louis, MO: WB. Saunders Co; 2004. p. 169-192.

58. Amang AP, Kodji E, Mezui C, Baane MP, Siwe GT, Kuissu TM, Emakoua J, Tan PV. Hepatoprotective Effects of Aqueous Extract of Opiliaceltidifolia (Opiliaceae) Leaves against Ethanol-Induced Liver Damage in Rats 2020; 8.

59. Irondi EA, Oboh G, Agboola O, Boligon AA, Athayde ML. Phenolics extract of Tetrapleura tetraptera fruit inhibits xanthine oxidase and $\mathrm{Fe}^{2+}$-induced lipid peroxidation in the kidney, liver, and lungs 
tissues of rats in vitro, Food Sci. Hum. Wellness. 2015; 5, 17-23.

60. Hussain T, Gupta RK, Sweety K, Eswaran B, Vijayakumar M, Venkateswara CR. Nephroprotective activity of Solanum xanthocarpum fruit extract against gentamicin-induced nephrotoxicity and renal dysfunction in experimental rodents. Asian Pac. J. Trop. Dis. 2012; 5, 686-691.

61. Seifa MM, Madbolib AN, Marreza DA, Aboulthanac WMK. Hepato-renal protective effects of Egyptian purslane extract against experimental cadmium toxicity in rats with special emphasis on the functional and histopathological changes. Toxicol. Rep. 2019; 6, 625-631.

62. Moukette MB, Pieme AC, Biapa PCN,Njimou JR, Stoller M, Bravi M, Je. In vitro ion chelating, antioxidative mechanism of extracts from fruits and barks of Tetrapleura tetraptera and their protective effects against fenton mediated toxicity of metal ions on liver homogenates. Evid. Based Complement. Alternat. Med. 2015, 423689.

63. Schaffer A, Menche N. Anatomie physiologie biologie. In: Edition Française traduite de la 4e edition allemande, 2e edn. Medecine/sciences, Paris, 2004; pp. 225-271.

64. MushtaqA, Naqvi SF, Anwar R, Mehwish J, Anwar H, Asifa B, Qurat-ul-Ain, Baderqa TA.Evaluation of hypolipidemic activity of Allium schoenoprasum in Albino Rats. Br. J. Pharmaceut. Res. 2017; 14, 110.

65. Nwozo SO, and OrojobiBF. Hypolipiemic and antioxidant effects of Tetrapleura tetraptera fruits, including seeds, in hypercholesterolaemic rats. Seed Sci. Biotechnol.2010; 4, 73-78.

\section{Figures}
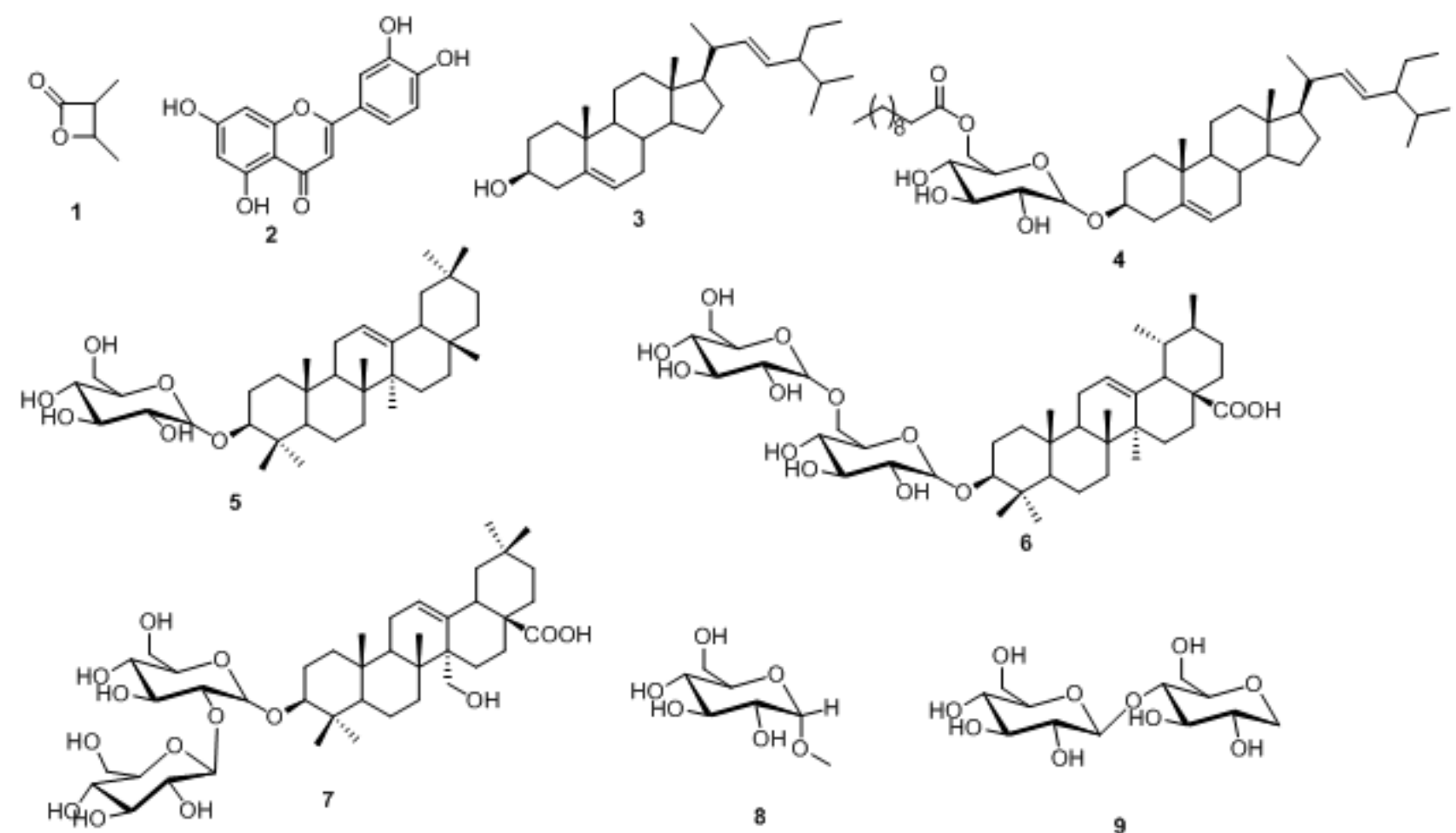

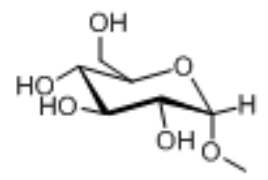

8

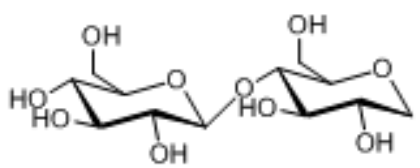

9

Figure 1 
Chemical structures of compounds 1 to 9 isolated from TTF extract.

1: (3R, 4S)-3,4-dimethyloxetan-2-one; 2: luteolin, 3: stigmasterol; 4; 3-O-[6'-O-undecanoyl- $\beta_{-D^{-}}$ glucopyranosyl] stigmasterol; 5: oleanan-12-en-3- $\beta-O_{-D}$-glucopyranoside; 6: 3- $O-\beta_{D}$-glucopyranosyl-(1 $\left.\rightarrow 6\right)$ $\beta_{D}$-glucopyranosylurs-12-en-28-oic acid; 7: 3- 0 - $\beta_{D}$-glucopyranosyl-(1 $\left.\rightarrow 3\right)-\beta_{D}$-glucopyranosyl-27-

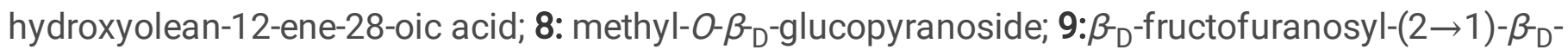
glucopyranoside
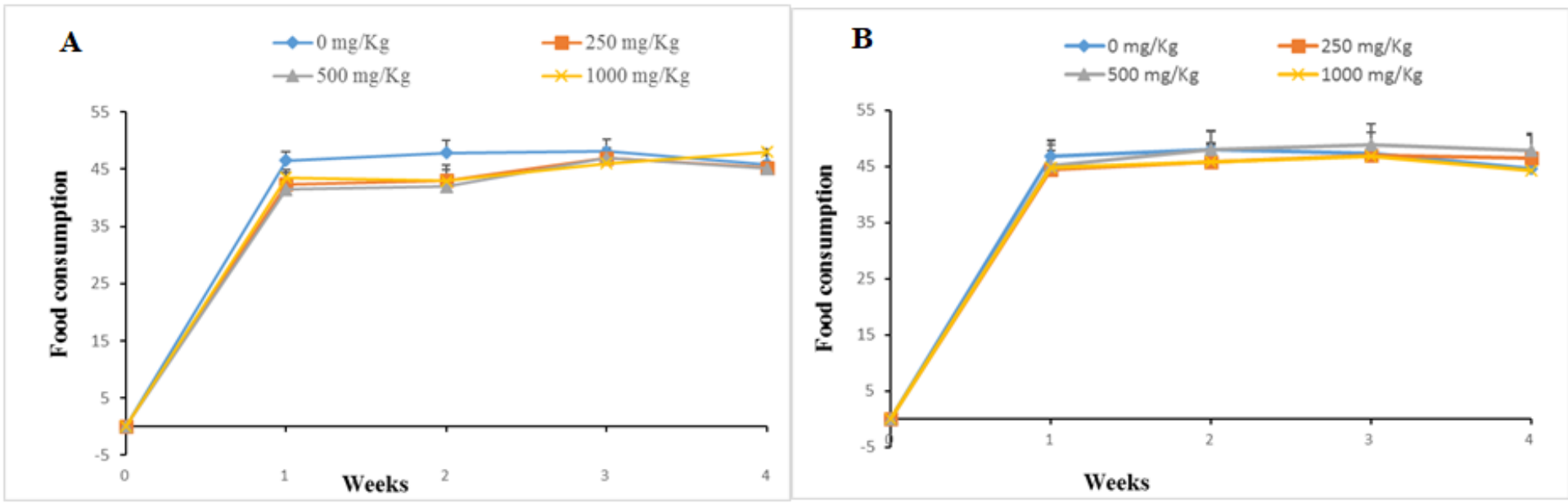

Figure 2

Effect of daily oral administration of TTF on food intake in female (A) and male (B) rats.

Animals received TTF (250, 500, and $1000 \mathrm{mg} / \mathrm{kg}, \mathrm{BW})$ and vehicle (5\% DMSO solution). All data are presented as mean $\pm S D$ for $n=4$ per group.
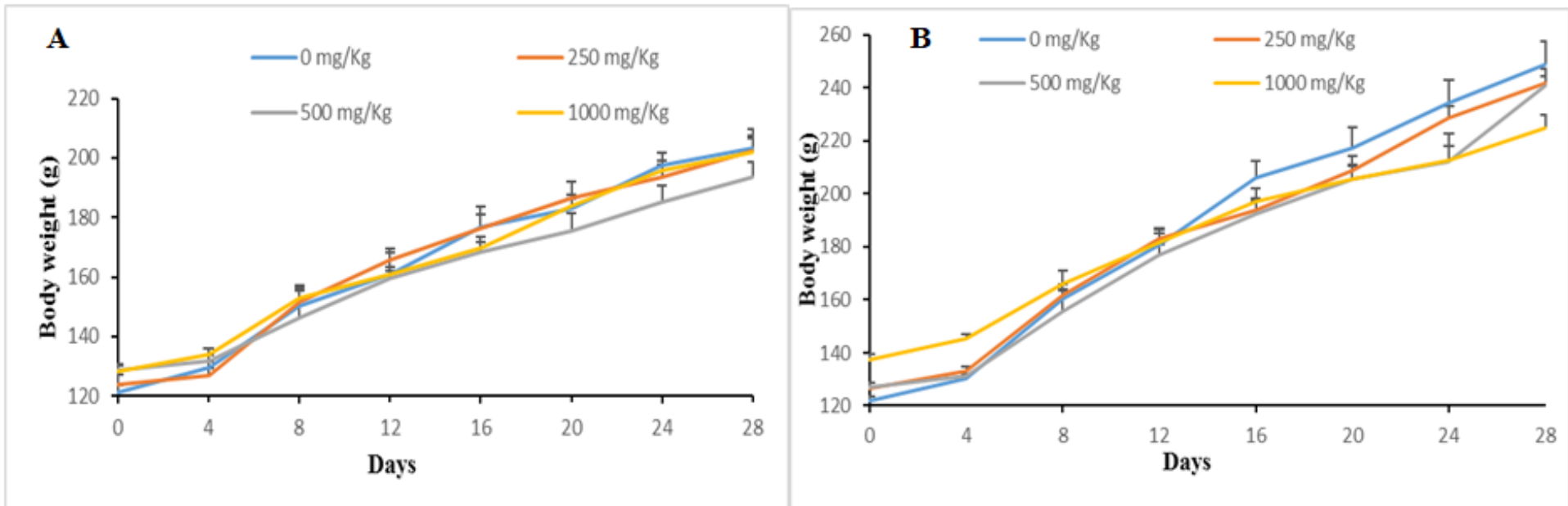

Figure 3 
Effect of daily oral administration of TTF on body weight changes in female (A) and male (B) rats.

Animals received TTF (250, 500, and $1000 \mathrm{mg} / \mathrm{kg}, \mathrm{BW})$ and vehicle (5\% DMSO solution). All data are presented as mean $\pm S D$ for $n=4$ per group.
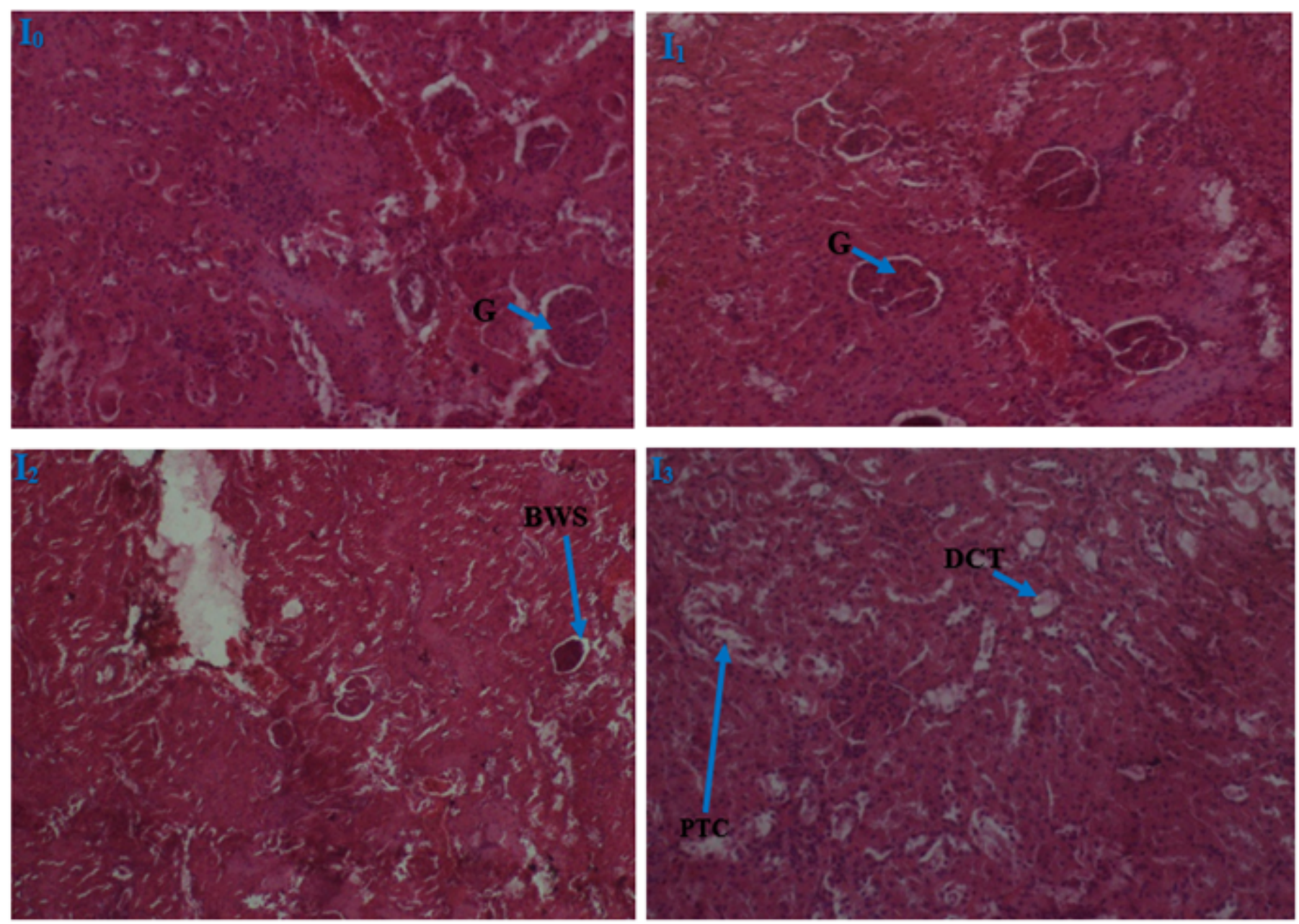

\section{Figure 4}

Histopathological changes in kidney of rats after 28 days of treatment in female rats (400x).

$\left(I_{0}\right)$ control; $\left(I_{1}\right)$ Wistar strain rats treated with $250 \mathrm{mg} / \mathrm{kg}$ TTF extract; $\left(I_{2}\right)$ Wistar strain rats treated with $500 \mathrm{mg} / \mathrm{kg}$ TTF extract; $\left(\mathrm{I}_{3}\right)$ Wistarstrain rats treated with $1000 \mathrm{mg} / \mathrm{kg}$ TTF extract; G: glomerulus, BWS: Bowman space, DCT: distal convoluted tubule, PTC: proximal convoluted tubule. 

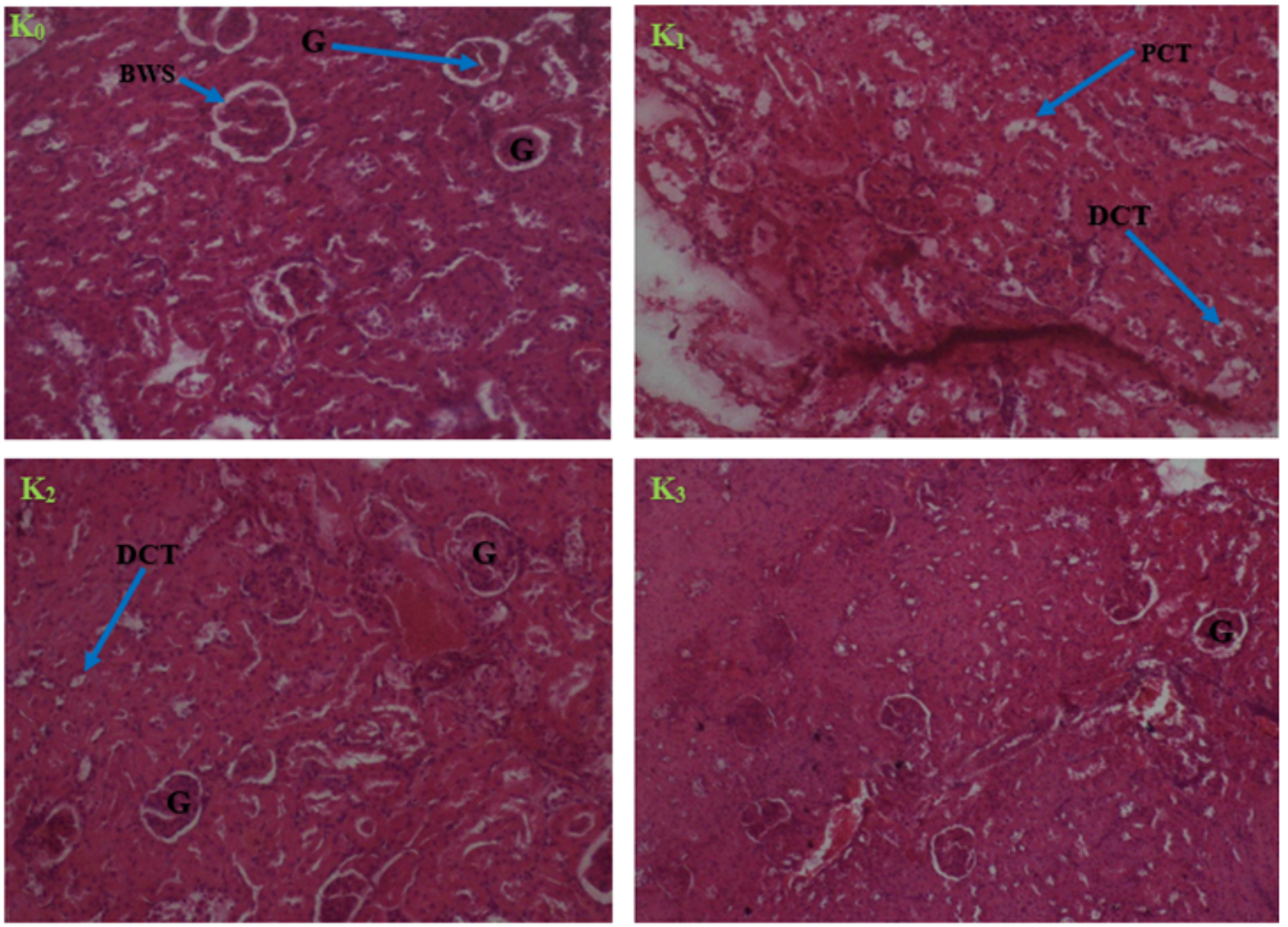

\section{Figure 5}

Histopathological changes in kidney of rats after 28 days of treatment in male rats (400x).

$\left(I_{0}\right)$ control; $\left(I_{1}\right)$ Wistar strain rats treated with $250 \mathrm{mg} / \mathrm{kg}$ TTF extract; $\left(I_{2}\right)$ Wistar strain rats treated with $500 \mathrm{mg} / \mathrm{kg} \mathrm{T}$; $\left(\mathbf{I}_{3}\right)$ Wistarstrain rats treated with $1000 \mathrm{mg} / \mathrm{kg}$ TTF extract; G: glomerulus, BWS: Bowman space, DCT: distal convoluted tubule, PTC: proximal convoluted tubule. 

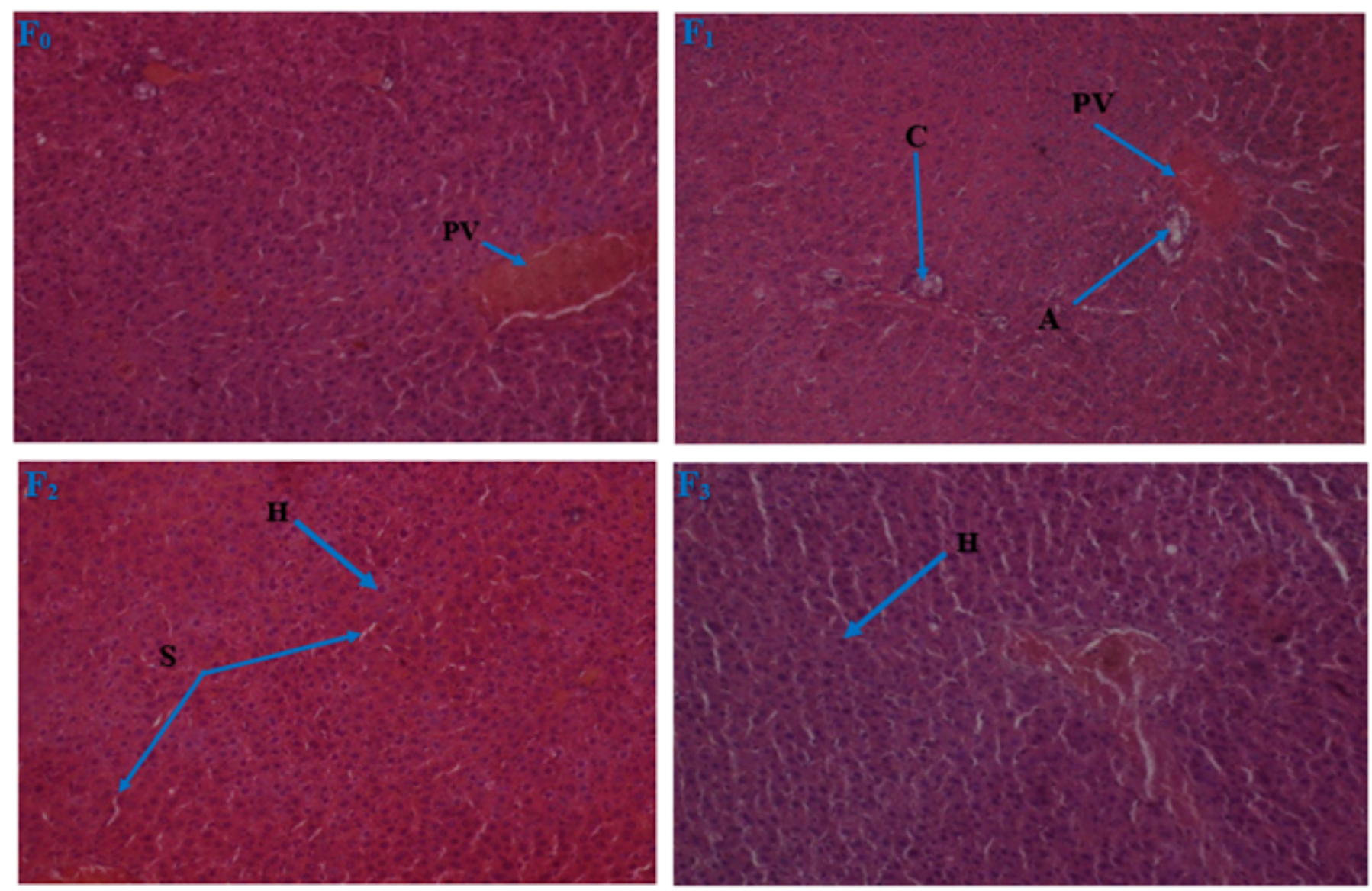

\section{Figure 6}

Histopathological changes in liver of rats after 28 days of treatment in female rats (400x).

$\left(F_{0}\right)$ control; $\left(F_{1}\right)$ Wistar strain rats treated with $250 \mathrm{mg} / \mathrm{kg}$ TTF extract; $\left(\boldsymbol{F}_{2}\right)$ Wistar strain rats treated with $500 \mathrm{mg} / \mathrm{kg}$ TTF extract; $\left(\boldsymbol{F}_{3}\right)$ Wistar strain rats treated with $1000 \mathrm{mg} / \mathrm{kg}$ TTF extract, PV: Hepatic portal vein, $\mathbf{H}$ : hepatocytes, C: Centrolobular vein, $\mathbf{S}$ : sinusoid, $\mathbf{A}$ : bile duct. 

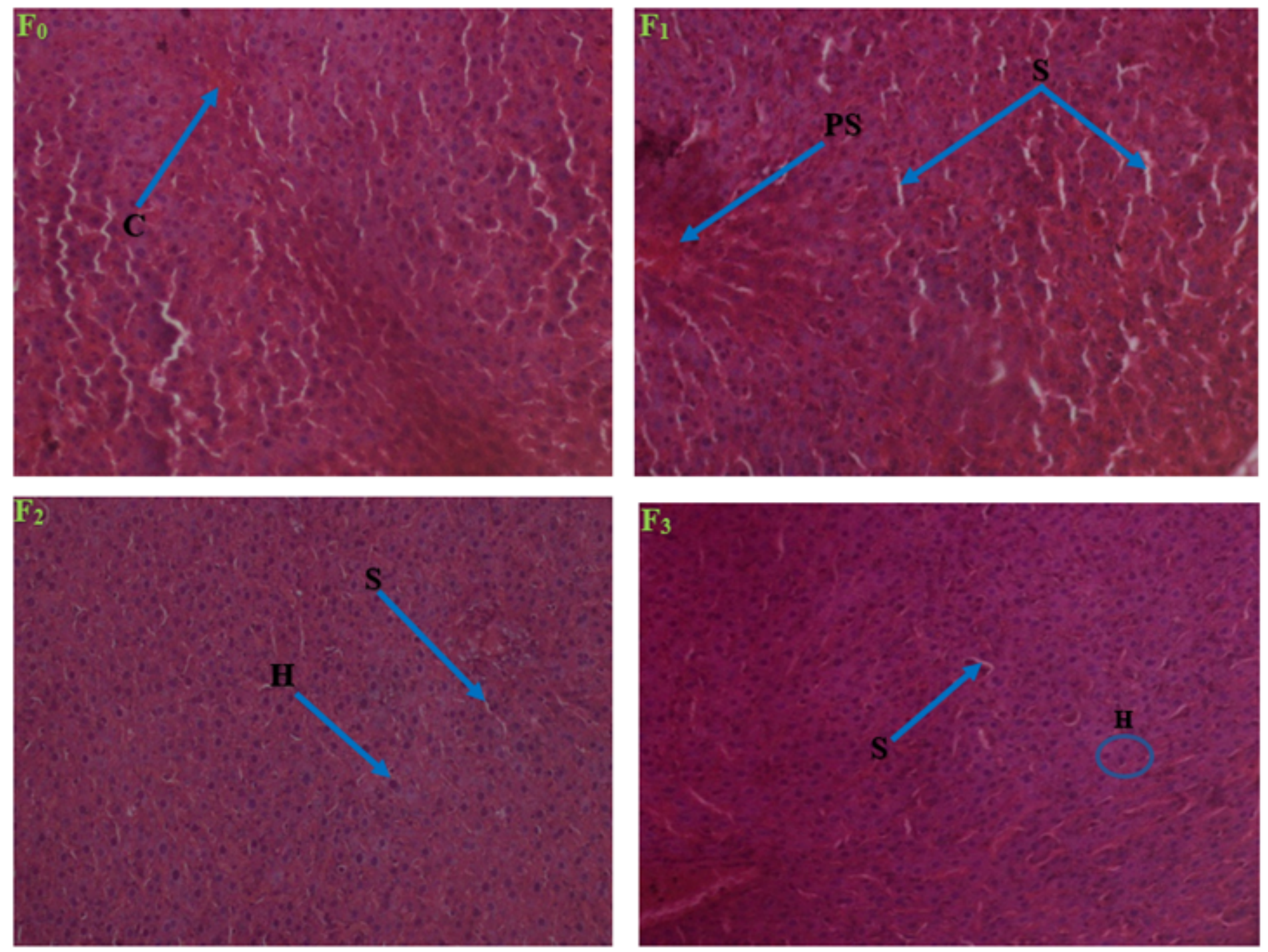

\section{Figure 7}

Liver sections showing the effect of $T$. tetraptera methanolic extract in 28-day subacute toxicity study in male rats.

$\left(F_{0}\right)$ : control group; $\left(F_{1}\right): 250 \mathrm{mg} / \mathrm{kg} ;\left(F_{2}\right): 500 \mathrm{mg} / \mathrm{kg}$ and $\left(F_{3}\right): 1000 \mathrm{mg} / \mathrm{kg}$. Indicators: PS: portal space; $\mathrm{H}$ : hepatocytes; C: centrolobular vein; (S): sinusoid; (Cb): bile duct.

\section{Supplementary Files}

This is a list of supplementary files associated with this preprint. Click to download.

- GuidelinesARRIVE.docx

- Supplementaryfile180222.docx 